\title{
American Options with Discontinuous Two-Level Caps
}

\author{
Jerome Detemple \& Yerkin Kitapbayev
}

19 July 2017

\begin{abstract}
This paper examines the valuation of American capped call options with two-level caps. The structure of the immediate exercise region is significantly more complex than in the classical case with constant cap. When the cap grows over time, making extensive use of probabilistic arguments and local time, we show that the exercise region can be the union of two disconnected set. Alternatively, it can consist of two sets connected by a line. The problem then reduces to the characterization of the upper boundary of the first set, which is shown to satisfy a recursive integral equation. When the cap decreases over time, the boundary of the exercise region has piecewise constant segments alternating with non-increasing segments. General representation formulas for the option price, involving the exercise boundaries and the local time of the underlying price process, are derived. An efficient algorithm is developed and numerical results are provided.
\end{abstract}

\section{Introduction}

Caps are attractive provisions found in a variety of derivative contracts. For an investor seeking to establish a short position, a cap limits the exposure to unfavorable price movements. For a buyer, it reduces the price, hence the cost of entering a long position. Products with caps can be found on organized exchanges, in OTC markets, or appear as components of financial packages offered by firms to raise capital. A common example is the CAPS contract, introduced in 1991 by the CBOE and traded to the end of the 90s. This option, which is written on the S\&P100 and S\&P500 indices, has gains limited to 30 -point moves in the underlying index. It also closes automatically, hence has an automatic exercise provision, once the change in the underlying index hits the cap. Another example is the Mexican Index-Linked Euro Security (MILES), which traded in the early 90s on the Luxembourg stock exchange. The MILES is an American capped option on the Mexican stock index, the Bolsa Mexicana de Valores. It has a initial vesting period during which exercise is prohibited, followed by a period during which exercise is at the option of the holder, but with gains limited by the cap provision. It is thus a special case of an American capped option with two-level cap. Nowadays, caps appear as features that can be incorporated in FLEX options offered by the CBOE. FLEX

Mathematics Subject Classification 2010. Primary 91G20,60G40. Secondary 60J60, 35R35, 45G10.

Key words and phrases: American capped option, optimal stopping, geometric Brownian motion, freeboundary problem, local time, integral equation 
options are fully customizable products written on indices or equities. They can be Europeanor American-style. Bull and Bear spreads are examples of FLEX options with caps.

The valuation of capped option has been a topic of long-standing interest in academic and practitioner circles. Early studies dealing with the valuation of European-style capped options include Boyle and Turnbull (1989). Capped options with automatic exercise at the cap are discussed by Flesacker (1992) and Chance (1994), among others. The case of American-style capped options is examined by Broadie and Detemple (1995). They consider both constant and growing caps. Using dominance arguments, they show that the optimal exercise policy for a capped call with maturity date $T$ and constant cap $L$ is the first hitting time of $B \wedge L$, the minimum of the cap $L$ and the optimal exercise boundary $B=(B(t))_{t \in[0, T]}$ of an uncapped, but otherwise identical option. They deduce several representations of the capped option price. For a cap that grows at a constant rate $g$, they show that the optimal policy lies in a 3parameter family. They characterize the optimal parametric policy and find the associated option value. In a recent study, Qiu (2015) analyzes American capped strangle options and exploits the local time-space formula to derive an early exercise premium (EEP) representation. The pair of optimal exercise boundaries is characterized as the unique solution to a system of coupled integral equations.

This paper is devoted to the pricing of an American capped call option with a two-level cap $L_{1} 1_{\tau<T_{1}}+L_{2} 1_{T_{1} \leq \tau \leq T_{2}}$, where $L_{1}, L_{2}$ are arbitrary levels such that $L_{1}, L_{2}>K$. Such a cap structure provides more flexibility from the point of view of an issuing firm seeking to raise capital. The case $L_{1}<L_{2}$ is especially relevant for the success of an issue, as the growing upside potential of the contract raises its attractiveness for potential buyers. At the same time, the two-tiered cap still limits the exposure of the firm, making it interesting for the issuer. Nevertheless, for completeness, we also consider the case $L_{2}<L_{1}$.

It is clear that on the interval $\left[T_{1}, T_{2}\right]$, the pricing problem reduces to the problem with single cap $L_{2}$ for which the immediate exercise region $\mathcal{E}_{2}$ was characterized by Broadie and Detemple (1995). Thus, $\mathcal{E}_{2}=\left\{(S, t) \in \mathbb{R}^{+} \times\left[T_{1}, T_{2}\right]: S \geq B(t) \wedge L_{2}\right\}$. We make use of the local time-space calculus of Peskir (2005) and provide an alternative representation for the option price using the European capped option as the benchmark for the early exercise premium formula. Due to the non-smoothness of the payoff function in the exercise region at the cap $L_{2}$, a local time term appears in the pricing formula. However, this representation is found to be efficient and we exploit it extensively for the analysis of the optimal exercise strategy on $\left[0, T_{1}\right)$. Remarkably, this representation also provides an alternative proof of the result of Broadie and Detemple (1995). Namely, it shows that the part of the boundary below the cap satisfies the same integral equation as the boundary of the American uncapped call option (see Remark 2.6).

We then consider the pricing problem over the interval $\left[0, T_{1}\right)$. There are three cases of interest. In the first case, $L_{1}<B\left(T_{1}\right) \wedge L_{2}$. We show that the immediate exercise region on $\left[0, T_{1}\right)$ takes the form

$$
\mathcal{E}_{1}=\left\{(S, t) \in \mathbb{R}^{+} \times\left[0, t^{1}\right]: L_{1} \leq S \leq B^{L, 1}(t)\right\}
$$

where $B^{L, 1}=\left(B^{L, 1}(t)\right)_{t \in\left[0, t^{1}\right]}$ is an upper boundary and $t^{1}<T_{1}$ is an endogenous time. It has three unusual features: (i) if $t^{0} \equiv T_{1}-\frac{1}{r} \log \left(\left(L_{2}-K\right) /\left(L_{1}-K\right)\right) \geq 0$, then $B^{L, 1}(t)=+\infty$ for $t \in\left[0, t^{0}\right]$ and $B^{L, 1}\left(t^{0}+\right)=+\infty$; (ii) there exists a time $t^{0} \vee 0 \leq t^{1}<T_{1}$ such that $(S, t) \notin \mathcal{E}_{1}$ for any $S>0$ and $t \in\left(t^{1}, T_{1}\right)$; (iii) there exists a time $t^{0} \vee 0 \leq T_{0} \leq t^{1}$ such that 
$B^{L, 1}(t)=L_{1}$ for $t \in\left[T_{0}, t^{1}\right]$. We characterize the times $\left(t^{0}, T_{0}, t^{1}\right)$ and show that the upper boundary $B^{L, 1}$ solves a recursive integral equation on $\left[t^{0} \vee 0, T_{0}\right]$.

In the second case, $B\left(T_{1}\right) \leq L_{1}<L_{2}$. The immediate exercise region on $\left[0, T_{1}\right)$ becomes

$$
\begin{aligned}
\mathcal{E}_{1}= & \left\{(S, t) \in \mathbb{R}^{+} \times\left[0, T_{1}\right): L_{1} \leq S \leq B^{L, 1}(t)\right\} \\
& \cup\left\{(S, t) \in \mathbb{R}^{+} \times\left[t^{*}, T_{1}\right): B(t) \leq S \leq L_{1}\right\}
\end{aligned}
$$

where $t^{*}$ is the unique solution to the equation $B(t)=L_{1}$ and the upper boundary $B^{L, 1}=$ $\left(B^{L, 1}(t)\right)_{t \in\left[0, T_{1}\right)}$ is defined over the whole interval $\left[0, T_{1}\right)$. Moreover, there exists $T_{0}<T_{1}$ such that $B^{L, 1}(t)=L_{1}$ for $t \in\left[T_{0}, T_{1}\right)$. The main differences with the previous case are that immediate exercise (i) is always optimal when $S=L_{1}$ (thus $t^{1}=T_{1}$ ) and (ii) is optimal below $L_{1}$ when $B(t) \leq S \leq L_{1}$. We derive the corresponding recursive integral equation for $B^{L, 1}$ on $\left[t^{0} \vee 0, T_{0}\right]$.

In the last case, when $L_{1}>L_{2}$, we assume that the cap is left-continuous. We impose this condition in order to avoid technical difficulties associated with the discontinuity of the option price at $T_{1}$ from the left and the possible non-existence of an optimal exercise time. Moreover, the prices of the options with left- and right-continuous caps coincide prior to $T_{1}$. For this case, we show that the immediate exercise region is

$$
\mathcal{E}=\left\{(S, t) \in \mathbb{R}^{+} \times\left[0, T_{1}\right]: S \geq B^{L, 1}(t)\right\} \cup\left\{(S, t) \in \mathbb{R}^{+} \times\left(T_{1}, T_{2}\right]: S \geq B(t) \wedge L_{2}\right\}
$$

where $B^{L, 1}=\left(B^{L, 1}(t)\right)_{t \in\left[0, T_{1}\right]}$ is an endogenous non-increasing boundary defined over $\left[0, T_{1}\right]$ satisfying the boundary condition $B^{L}\left(T_{1}-\right)=\max \left(r K / \delta, L_{2} \wedge B\left(T_{1}\right)\right)$ and $B^{L}\left(T_{1}\right)=L_{2} \wedge$ $B\left(T_{1}\right)$. In this instance, immediate exercise above the cap $L_{1}$ is always optimal on $\left[0, T_{1}\right)$. Immediate exercise below the cap is also optimal if $B^{L, 1}(t) \leq S \leq L_{1}$. We derive the recursive integral equation for $B^{L, 1}$ on $\left[0, T_{1}\right)$.

In all cases, the immediate exercise region over the whole interval $\left[0, T_{2}\right]$ is the union of the two sets, $\mathcal{E}_{1}=\mathcal{E}_{1} \cup \mathcal{E}_{2}$. In the first case described above, the sets in question are disconnected, i.e., they are separated by a region in which continuation is optimal. For each case, we derive a general representation formula for the option price, involving the relevant boundary and the local times of the underlying price process at the cap and the boundary. We also develop an efficient algorithm for computing the exercise boundary and the option price, and provide numerical results.

The main difficulties for pricing this option and determining the optimal exercise policy, compared to the standard American-style derivatives, are the non-smoothness of the payoff with respect to underlying asset price in the exercise region and the time-discontinuity of the payoff. Although the first issue can be tackled as in aforementioned papers, the second feature induces a complicated structure of the exercise region and prevents the application of standard methods. This paper provides probabilistic arguments, making extensive use of dominance relations and local time to tackle these types of difficulties. Other potential applications of our approach include finite horizon impulse control problems in the presence of execution delays and decision lags, which are common in algorithmic trading and other decision-making processes. For related studies and theoretical background, we refer to Palczewski and Stettner (2010), among others, who showed regularity of the value functions of optimal stopping problems with time-discontinuous payoffs under a quite general class of Markov processes. 
The paper is organized as follows. Section 2 formulates the problem, revisits the results of Broadie and Detemple (1995) and provides an alternative pricing formula for the constant cap option problem. Section 3 contains the main result of the paper. It describes the optimal exercise policy for the three cases of interest. Sections 4, 5 and 6 consider each case in isolation and prove the optimality of the exercise policy announced in Section 3. They also provide the relevant early exercise premium formulas for the option price. Finally, Section 7 describes a step-by-step algorithm for computation and presents numerical results.

\section{Problem Formulation and Preliminary Results}

Consider the probability space $(\Omega, \mathcal{F}, Q)$ where $Q$ is the risk-neutral measure. Let $W$ be a $Q$-standard Brownian motion with natural filtration $\left(\mathcal{F}_{t}\right)_{t>0}$. We assume the standard financial market with an underlying asset price $S$ that follows a geometric Brownian motion under the risk-neutral measure $Q$

$$
d S_{t}=S_{t}\left((r-\delta) d t+\sigma d W_{t}\right)
$$

where $r>0$ is the interest rate, $\delta \geq 0$ the dividend yield, and $\sigma>0$ the volatility parameter.

An American capped call option has exercise payoff $\left(S_{\tau} \wedge L(\tau)-K\right)^{+}$at exercise time $\tau$ where $L=(L(t))_{t \in\left[0, T_{2}\right]}$ is the cap. In this paper, we focus on capped options with two-level caps, i.e., such that

$$
L(\tau)=L_{1} 1_{\tau<T_{1}}+L_{2} 1_{T_{1} \leq \tau \leq T_{2}}
$$

with $T_{1}<T_{2}$. The cap $L(t)$ is right-continuous with jump at the intermediate date $T_{1}$ if $L_{1} \neq L_{2}$. If $L_{1}>L_{2}$, we restrict attention to the left-continuous version of this cap, namely $L(\tau)=L_{1} 1_{\tau \leq T_{1}}+L_{2} 1_{T_{1}<\tau \leq T_{2}}$. The strike price is $K$ and the maturity date $T_{2}$. To avoid trivial cases we assume $L_{1}, L_{2}>K$.

Standard arguments (e.g., Karatzas (1988)) can be invoked to write the price function as

$$
C^{A, L}(S, t)=\sup _{t \leq \tau \leq T_{2}} \mathrm{E}_{t}\left[e^{-r(\tau-t)}\left(S_{\tau} \wedge L(\tau)-K\right)^{+}\right]
$$

at time $0 \leq t \leq T_{2}$ and given the stock price $S>0$, where the supremum is taken over all stopping times $\tau \in\left[t, T_{2}\right]$ w.r.t. the filtration $\left(\mathcal{F}_{t}\right)_{t \geq 0}$. Throughout the paper, we use the notation $\mathrm{E}_{t}[\cdot] \equiv \mathrm{E}\left[\cdot \mid S_{t}=S\right]$ and $\mathrm{E}$ is the expectation under $Q$. We also define the infinitesimal generator of $S$ as

$$
\mathbb{L}_{S}=(r-\delta) S \frac{\partial}{\partial S}+\frac{\sigma^{2}}{2} S^{2} \frac{\partial^{2}}{\partial S^{2}}
$$

We note that it is not true in general that the value functions of optimal stopping problems with discontinuous payoffs are continuous and that optimal stopping rules have the standard form. However, if $L_{1}<L_{2}$ we can apply Theorem 3.1 iii) of Palczewski and Stettner (2010) to obtain that $C^{A, L}$ is continuous and the optimal exercise time is given as

$$
\tau^{*}(t)=\inf \left\{u \geq t: C^{A, L}\left(S_{u}, u\right)=\left(S_{u} \wedge L(u)-K\right)^{+}\right\} \wedge T_{2} .
$$


for $t \leq T_{2}$. The case $L_{1}>L_{2}$ is more complicated as the value function becomes discontinuous at $T_{1}$ from the left and we may have non-existence of an optimal stopping time. To avoid these issues, we will consider the left-continuous cap $L(\tau)=L_{1} 1_{\tau \leq T_{1}}+L_{2} 1_{T_{1}<\tau \leq T_{2}}$ when $L_{1}>L_{2}$ and show the connection between two versions (see Section 6).

Let $B=(B(t))_{t \in\left[0, T_{2}\right]}$ denote the optimal exercise boundary of an uncapped call option with identical strike $K$ and maturity date $T_{2}$, i.e., with exercise payoff $\left(S_{\tau}-K\right)^{+}$. In the case $L_{1}<L_{2}$, following Broadie and Detemple (1995), we define $t^{*}$ as follows

$$
\left\{\begin{array}{l}
t^{*}=s, \quad \text { if } B(s)=L(s) \text { for some } s \in\left[0, T_{2}\right] \\
t^{*}=0, \quad \text { if } B(\cdot)<L(\cdot) \text { on } \in\left[0, T_{2}\right] \\
t^{*}=T_{2}, \quad \text { if } B\left(T_{2}-\right)>L_{2} \\
t^{*}=T_{1}, \quad \text { if } L_{1}<B\left(T_{1}\right)<L_{2} .
\end{array}\right.
$$

Thus, $t^{*}$ is the unique solution to the equation $B(s)=L(s)$, if such a solution exists. Otherwise, $t^{*}$ is at a corner of the interval $\left[0, T_{2}\right]$. It is clear that for $t \in\left[T_{1}, T_{2}\right]$, the optimal exercise rule follows the strategy for the capped option $C^{A, L_{2}}$ with single cap $L_{2}$. This problem has been solved in Broadie and Detemple (1995) and, in Theorems 2.1 and 2.2 below, we revisit their results.

Theorem 2.1. The optimal exercise boundary of the capped call option (2.3) on $\left[T_{1}, T_{2}\right]$ is $B^{L, 2}=B \wedge L_{2}$ so that the optimal exercise time in (2.3) is given by

$$
\tau^{*}(t)=\inf \left\{u \geq t: S_{u} \geq B^{L, 2}(u)\right\} \wedge T_{2}
$$

for $t \in\left[T_{1}, T_{2}\right]$. The price of the capped call (2.3) is then

$$
C^{A, L_{2}}(S, t)=\mathrm{E}_{t}\left[e^{-r\left(\tau^{*}(t)-t\right)}\left(S_{\tau^{*}(t)} \wedge L_{2}-K\right)^{+}\right]
$$

for $S>0$ and $t \in\left[T_{1}, T_{2}\right]$.

Figure 1 illustrates the optimal exercise boundary $B^{L, 2}=B \wedge L_{2}$ for the single cap problem. The optimal exercise region $\mathcal{E}_{2}$ for the problem (2.3) when time is restricted to the interval $\left[T_{1}, T_{2}\right]$, is then clearly

$$
\mathcal{E}_{2}=\left\{(S, t) \in \mathbb{R}^{+} \times\left[T_{1}, T_{2}\right]: S \geq B^{L, 2}(t)\right\} .
$$

Using the theorem above, Broadie and Detemple (1995) derived a pricing formula for $C^{A, L_{2}}\left(S, T_{1}\right)$ by noting that if $t_{*}>T_{1}$ then $S$ either hits $L_{2}$ before $t^{*}$ or the capped option becomes essentially the uncapped call at $t^{*}$. If $t^{*} \leq T_{1}$, then for $S<L_{2}$ the capped option price coincides with the uncapped option price. An alternative formula was obtained by using a capped option $C^{a e, L_{2}}$ with automatic exercise at $L_{2}$ as the benchmark for an early exercise premium representation.

Theorem 2.2. ( $i)$ For $t \in\left[T_{1}, T_{2}\right]$, the price of the capped call option with discontinuous cap $L$ is equal to the capped call option price with constant cap $L_{2}$ so that

$$
C^{A, L}(S, t)=C^{A, L_{2}}(S, t)
$$




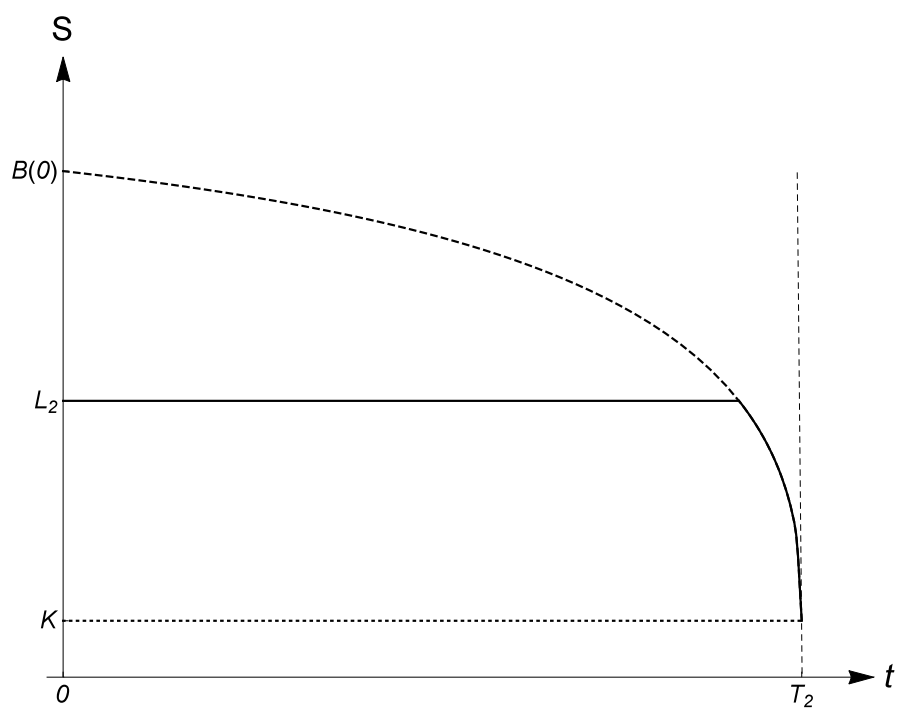

Figure 1. This figure plots the optimal exercise boundary $B^{L, 2}=B \wedge L_{2}$ for the single cap option problem. The dashed curve represents the uncapped exercise boundary $B$. The parameter set is $T_{2}=4, K=1, L_{2}=1.39, r=0.1, \delta=0.1, \sigma=$ 0.3 .

$$
=\left(L_{2}-K\right) \mathrm{E}_{t}\left[e^{-r\left(\tau^{*}(t)-t\right)} 1_{\left\{\tau^{*}(t)<t^{*}\right\}}\right]+\mathrm{E}_{t}\left[e^{-r\left(t^{*} \vee t-t\right)} C^{A}\left(S_{t^{*} \vee t}, t^{*} \vee t\right) 1_{\left\{\tau^{*}(t) \geq t^{*}\right\}}\right]
$$

for $S>0$, where $C^{A}$ is the price of a standard American call option with strike $K$ and maturity $T_{2}$. The second term can be simplified using the known distribution of $\tau^{*}(t)$ on $\left[t, t^{*} \vee t\right]$ (i.e., first hitting time of a constant barrier) and is then given in terms of an integral of $C^{A}$. Lemma 8.1 in Appendix shows how to compute both expectations.

(ii) Alternatively, the capped option can be priced as

$$
C^{A, L_{2}}(S, t)=C^{a e, L_{2}}(S, t)+\mathrm{E}_{t}\left[\int_{t}^{\tau_{L_{2}}} e^{-r(u-t)}\left(\delta S_{u}-r K\right) 1_{\left\{S_{u} \geq B(u)\right\}} d u\right]
$$

for $S>0$ and $t \in\left[T_{1}, T_{2}\right]$, where $C^{a e, L_{2}}(S, t)=\mathrm{E}_{t}\left[e^{-r\left(\tau_{L_{2}}-t\right)}\left(S_{\tau_{L_{2}}} \wedge L_{2}-K\right)^{+}\right]$is the price of a capped option $C^{a e, L_{2}}$ with automatic exercise at cap $L_{2}$ and $\tau_{L_{2}}=\inf \left\{u \geq t: S_{u}=\right.$ $\left.L_{2}\right\} \wedge T_{2}$ is the first hitting time of $L_{2}$ on $\left[t, T_{2}\right]$. The option price $C^{a e, L_{2}}$ can be computed in the closed form.

Formula (2.9) requires the integration of the standard American call option price $C^{A}\left(S, t^{*}\right)$ with respect to $S$, whereas the formula (2.10) boils down to the integration of the early exercise premium with respect to the joint distribution of $\tau_{L_{2}}$ and $S$. In this paper, we will make use of a third version of the capped option price, which is computationally convenient for our purposes. It is also based on an early exercise premium decomposition where the benchmark, in contrast, is given by the European capped option price $C^{E, L_{2}}(S, t)=\mathrm{E}_{t}\left[e^{-r\left(T_{2}-t\right)}\left(S_{T_{2}} \wedge L_{2}-K\right)^{+}\right]$. The proof relies on the local time-space formula on curves (Peskir (2005)). The noteworthy feature of the American capped option is that the payoff function $\left(S \wedge L_{2}-K\right)^{+}$is not smooth 


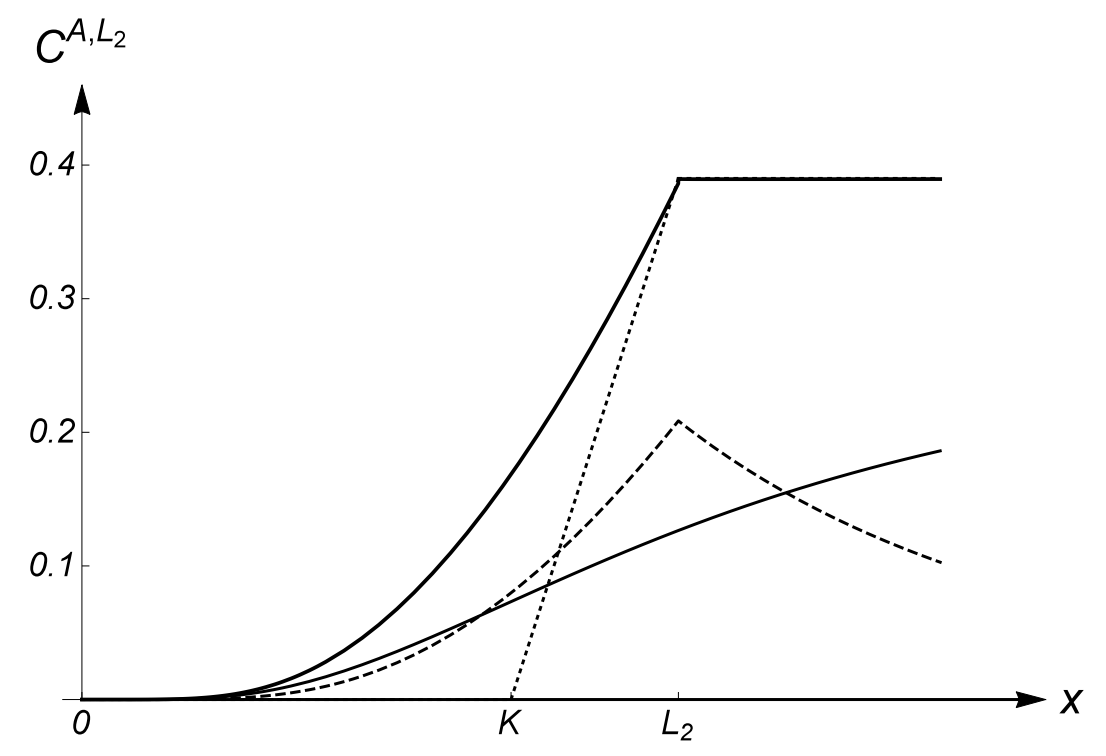

Figure 2. This figure plots the American capped option price $C^{A, L_{2}}(S, 0)$ with the constant cap $L_{2}$ (thick line). The thin line represents the European capped option price $C^{E, L_{2}}(S, 0)$, the dashed line represents the local time contribution, the dotted line is the immediate payoff $\left(S \wedge L_{2}-K\right)^{+}$. The parameter set is $T_{2}=4, K=1, L_{2}=1.39, r=0.1, \delta=0.1, \sigma=0.3$. It can be seen that the local time term has substantial value.

in the exercise region $\mathcal{E}_{2}$ when $S=L_{2}$. This fact implies the presence of a local time term in the option pricing formula (a similar result is obtained by Qiu (2015) for American capped strangle options). Figure 2 illustrates the American capped option price $C^{A, L_{2}}(S, 0)$ with the constant cap $L_{2}$. It can be clearly seen that the local time term brings significant value.

Theorem 2.3. The price of the American capped option $C^{A, L_{2}}(S, t)$ is

$$
\begin{aligned}
C^{A, L_{2}}(S, t)= & C^{E, L_{2}}(S, t)+r\left(L_{2}-K\right) \mathrm{E}_{t}\left[\int_{t}^{T_{2}} e^{-r(u-t)} 1_{\left\{S_{u} \geq L_{2}\right\}} d u\right] \\
& +\mathrm{E}_{t}\left[\int_{t}^{T_{2}} e^{-r(u-t)}\left(\delta S_{u}-r K\right) 1_{\left\{B(u) \leq S_{u} \leq L_{2}\right\}} d u\right] \\
& +\frac{1}{2} \mathrm{E}_{t}\left[\int_{t}^{T_{2}} e^{-r(u-t)} C_{S}^{A, L_{2}}\left(L_{2}-, u\right) d \ell_{u}^{L_{2}}\right]
\end{aligned}
$$

for $t \in\left[T_{1}, T_{2}\right]$ and $S>0$, where $\ell^{L_{2}}=\left(\ell_{t}^{L_{2}}\right)_{t \in\left[T_{1}, T_{2}\right]}$ is the local time that the process $S$ spends at $L_{2}$

$$
\ell_{t}^{L_{2}}=Q-\lim _{\varepsilon \downarrow 0} \frac{1}{2 \varepsilon} \int_{T_{1}}^{t} 1_{\left\{L_{2}-\varepsilon<S_{u}<L_{2}+\varepsilon\right\}} d\langle S\rangle_{u} .
$$

Proof. First, let us define

$$
\mathcal{C}^{L_{2}}=\left\{(S, t) \in \mathbb{R}^{+} \times\left[T_{1}, T_{2}\right): S<B^{L, 2}(t)\right\}
$$




$$
\begin{aligned}
& \mathcal{D}_{1}^{L_{2}}=\left\{(S, t) \in \mathbb{R}^{+} \times\left[T_{1}, T_{2}\right): S>L_{2}\right\} \\
& \mathcal{D}_{2}^{L_{2}}=\left\{(S, t) \in \mathbb{R}^{+} \times\left[t^{*} \vee T_{1}, T_{2}\right]: B^{L, 2}(t)=B(t)<S<L_{2}\right\}
\end{aligned}
$$

where $\mathcal{C}^{L_{2}}$ is the continuation set and $\mathcal{D}_{1}^{L_{2}}, \mathcal{D}_{2}^{L_{2}}$ are two parts of the exercise region. We also define the local time processes $\ell^{B^{L, 2}}=\left(\ell_{t}^{B^{L, 2}}\right)_{t \in\left[T_{1}, T_{2}\right]}$ and $\ell^{B}=\left(\ell_{t}^{B}\right)_{t \in\left[t^{*} \vee T_{1}, T_{2}\right]}$ at $B^{L, 2}$ on $\left[T_{1}, T_{2}\right]$ and at $B$ on $\left[t^{*} \vee T_{1}, T_{2}\right]$, respectively,

$$
\begin{aligned}
& \ell_{t}^{B^{L, 2}}=Q-\lim _{\varepsilon \downarrow 0} \frac{1}{2 \varepsilon} \int_{T_{1}}^{t} 1_{\left\{B^{L, 2}(u)-\varepsilon<S_{u}<B^{L, 2}(u)+\varepsilon\right\}} d\langle S\rangle_{u} \\
& \ell_{t}^{B}=Q-\lim _{\varepsilon \downarrow 0} \frac{1}{2 \varepsilon} \int_{t^{*} \vee T_{1}}^{t} 1_{\left\{B(u)-\varepsilon<S_{u}<B(u)+\varepsilon\right\}} d\langle S\rangle_{u} .
\end{aligned}
$$

Now, we verify that $C^{A, L_{2}}$ and $B^{L, 2}$ satisfy the conditions of the local time-space formula on curves (Peskir (2005), see Theorem 3.1 \& Remark 3.2). Indeed, (i) $C^{A, L_{2}}$ is $C^{1,2}$ in $\mathcal{C}^{L_{2}}$ (by strong Markov property) and is smooth in both $\mathcal{D}_{1}^{L_{2}}$ and $\mathcal{D}_{2}^{L_{2}}$; (ii) $B^{L, 2}$ and $L_{2}$ are continuous and of bounded variation as the former is non-increasing and the latter is a constant threshold; (iii) $Q\left(X_{t}=B^{L, 2}(t)\right)=0$ and $Q\left(X_{t}=L_{2}\right)=0$ for any $t \in\left[T_{1}, T_{2}\right]$ as $X$ is GBMP; (iv) $C_{t}^{A, L_{2}}+\mathrm{七}_{S} C^{A, L_{2}}-r C^{A, L_{2}}$ is locally bounded in $\mathcal{C}^{L_{2}} \cup \mathcal{D}_{1}^{L_{2}} \cup \mathcal{D}_{2}^{L_{2}}$ as it is zero in $\mathcal{C}^{L_{2}}$, and as $C^{A, L_{2}}(S, t)=L_{2}-K$ in $\mathcal{D}_{1}^{L_{2}}$ and $C^{A, L_{2}}(S, t)=S-K$ in $\mathcal{D}_{2}^{L_{2}}$; (v) $C^{A, L_{2}}$ is convex on $\mathcal{C}^{L_{2}}, \mathcal{D}_{1}^{L_{2}}$, and $\mathcal{D}_{2}^{L_{2}}$, respectively; (vi) $t \mapsto C_{S}^{A, L_{2}}\left(t, L_{2}+\right)=0$ and $t \mapsto C_{S}^{A, L_{2}}\left(t, B^{L, 2}(t)-\right.$ ) are continuous on $\left[T_{1}, T_{2}\right]$ (the latter holds due to the $C^{1,2}$-property of $C^{A, L_{2}}$ in $\left.\mathcal{C}^{L_{2}}\right)$; (vii) $t \mapsto C_{S}^{A, L_{2}}\left(t, L_{2}-\right)=1$ is continuous on $\left[t^{*} \vee T_{1}, T_{2}\right]$.

We can then apply Peskir's formula for $e^{-r\left(T_{2}-t\right)} C^{A, L_{2}}\left(S_{T_{2}}, T_{2}\right)$, implying that

$$
\begin{aligned}
& e^{-r\left(T_{2}-t\right)} C^{A, L_{2}}\left(S_{T_{2}}, T_{2}\right) \\
&=C^{A, L_{2}}(S, t)+\int_{t}^{T_{2}} e^{-r(u-t)}\left(C_{t}^{A, L_{2}}+\mathrm{七}_{S} C^{A, L_{2}}-r C^{A, L_{2}}\right)\left(S_{u}, u\right) d u+M_{T_{2}} \\
&+ \frac{1}{2} \int_{t}^{T_{2}} e^{-r(u-t)}\left(C_{S}^{A, L_{2}}\left(S_{u}+, u\right)-C_{S}^{A, L_{2}}\left(S_{u}-, u\right)\right) 1_{\left\{S_{u}=B^{L, 2}(u)\right\}} d \ell_{u}^{B^{L, 2}} \\
&+\frac{1}{2} \int_{t^{*} \vee t}^{T_{2}} e^{-r(u-t)}\left(C_{S}^{A, L_{2}}\left(S_{u}+, u\right)-C_{S}^{A, L_{2}}\left(S_{u}-, u\right)\right) 1_{\left\{S_{u}=L_{2}\right\}} d \ell_{u}^{L_{2}} \\
&= C^{A, L_{2}}(S, t)+M_{T_{2}}-r\left(L_{2}-K\right) \int_{t}^{T_{2}} e^{-r(u-t)} 1_{\left\{S_{u} \geq L_{2}\right\}} d u \\
&+\int_{t}^{T_{2}} e^{-r(u-t)}\left(r K-\delta S_{u}\right) 1_{\left\{B(u) \leq S_{u} \leq L_{2}\right\}} d u \\
&+\frac{1}{2} \int_{t}^{t^{*} \vee t} e^{-r(u-t)}\left(C_{S}^{A, L_{2}}\left(S_{u}+, u\right)-C_{S}^{A, L_{2}}\left(S_{u}-, u\right)\right) 1_{\left\{S_{u}=L_{2}\right\}} d \ell_{u}^{L_{2}} \\
&+\frac{1}{2} \int_{t^{*} \vee t}^{T_{2}} e^{-r(u-t)}\left(C_{S}^{A, L_{2}}\left(S_{u}+, u\right)-C_{S}^{A, L_{2}}\left(S_{u}-, u\right)\right) 1_{\left\{S_{u}=B(u)\right\}} d \ell_{u}^{B} \\
&+\frac{1}{2} \int_{t^{*} \vee t}^{T_{2}} e^{-r(u-t)}\left(C_{S}^{A, L_{2}}\left(S_{u}+, u\right)-C_{S}^{A, L_{2}}\left(S_{u}-, u\right)\right) 1_{\left\{S_{u}=L_{2}\right\}} d \ell_{u}^{L_{2}} \\
&= C^{A, L_{2}}(S, t)+M_{T_{2}}-r\left(L_{2}-K\right) \int_{t}^{T_{2}} e^{-r(u-t)} 1_{\left\{S_{u} \geq L_{2}\right\}} d u \\
&
\end{aligned}
$$




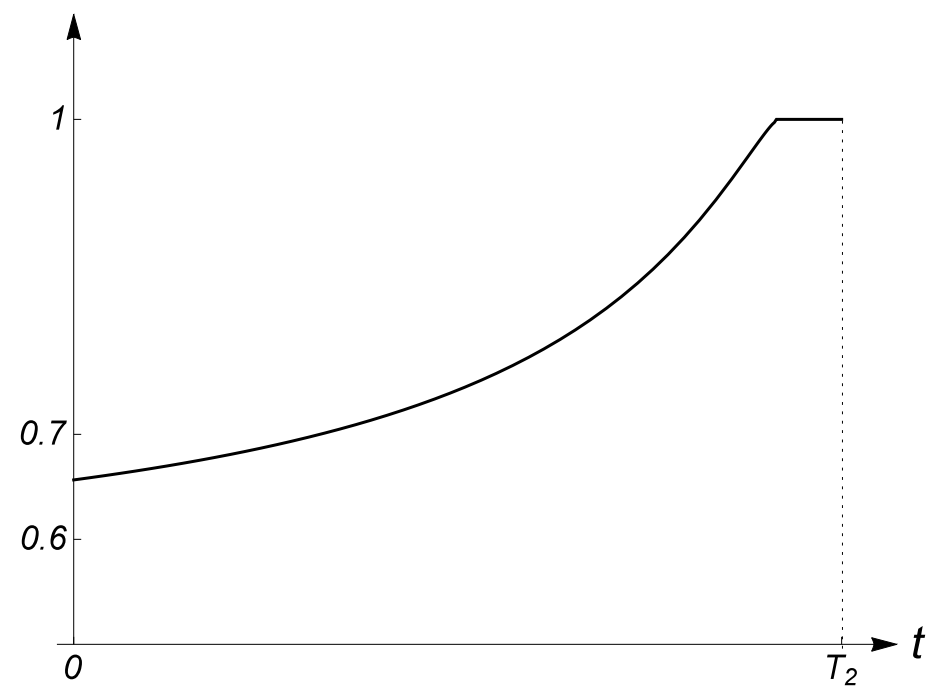

Figure 3. This figure plots the left-hand side derivative $C_{S}^{A, L_{2}}\left(L_{2}-, t\right)$ of $C^{A, L_{2}}$ at $L_{2}$ for $t \in\left[0, T_{2}\right)$. The parameter set is $T_{2}=4, K=1, L_{2}=1.39, r=0.1, \delta=$ $0.1, \sigma=0.3$.

$$
+\int_{t}^{T_{2}} e^{-r(u-t)}\left(r K-\delta S_{u}\right) 1_{\left\{B(u) \leq S_{u} \leq L_{2}\right\}} d u-\frac{1}{2} \int_{t}^{T_{2}} e^{-r(u-t)} C_{S}^{A, L_{2}}\left(L_{2}-, u\right) d \ell_{u}^{L_{2}}
$$

where $M=\left(M_{t}\right)_{t \geq T_{1}}$ is the martingale part and we used that $C_{t}^{A, L_{2}}+\mathrm{E}_{S} C^{A, L_{2}}-r C^{A, L_{2}}=0$ in $\mathcal{C}^{L_{2}} ; C^{A, L_{2}}(S, t)=L_{2}-K$ in $\mathcal{D}_{1}^{L_{2}}$ and $C^{A, L_{2}}(S, t)=S-K$ in $\mathcal{D}_{2}^{L_{2}} ;$ splitting the local time term w.r.t $\ell^{B^{L, 2}}$ into two parts; the smooth-fit condition at $B$ on $\left[t^{*} \vee T_{1}, T_{2}\right]$; and finally that $C_{S}^{A, L_{2}}\left(L_{2}+, \cdot\right)=0$. Now upon taking the expectation $\mathrm{E}_{t}$, using the optional sampling theorem for $M$, rearranging terms and noticing that $C^{A, L_{2}}\left(S, T_{2}\right)=\left(S \wedge L_{2}-K\right)^{+}$for all $S>0$, we get (2.11).

Remark 2.4. Using the definition of the local time and by straightforward calculations one can obtain that

$$
d \mathrm{E}_{t}\left[\ell_{u}^{L_{2}}\right]=\varphi\left(-\frac{\log \left(L_{2} / S\right)-\left(r-\delta-\frac{\sigma^{2}}{2}\right)(u-t)}{\sigma \sqrt{u-t}}\right) \frac{\sigma L_{2}}{\sqrt{u-t}} d u
$$

where $\varphi(z)=\frac{1}{\sqrt{2 \pi}} e^{-\frac{z^{2}}{2}}$ is the probability density function of the standard normal law, and thus the last term in (2.11) can be rewritten by inserting the expectation inside so that it becomes a Lebesgue-Stieltjes integral with respect to the expected local time

$$
\frac{1}{2} \int_{t}^{T_{2}} e^{-r(u-t)} C_{S}^{A, L_{2}}\left(L_{2}-, u\right) \varphi\left(-\frac{\log \left(L_{2} / S\right)-\left(r-\delta-\frac{\sigma^{2}}{2}\right)(u-t)}{\sigma \sqrt{u-t}}\right) \frac{\sigma L_{2}}{\sqrt{u-t}} d u .
$$

Remark 2.5. We note that formula (2.11) for $C^{A, L_{2}}$ is implicit and requires the knowledge of $C_{S}^{A, L_{2}}\left(L_{2}-, u\right)$, which can be estimated from the formula (2.9) for $u \in\left[T_{1}, t^{*}\right)$ and is given by 
$C_{S}^{A, L_{2}}\left(L_{2}-, u\right)=1$ for $u \in\left[t^{*}, T_{2}\right)$ (see Figure 3). This way of computing $C^{A, L_{2}}$ was found to be quite efficient as it does not use formula (2.9) globally, but only below $L_{2}$, to estimate the left derivative $C_{S}^{A, L_{2}}\left(L_{2}-, u\right)$.

Remark 2.6. If we take $t \geq\left[t^{*} \vee T_{1}, T_{2}\right]$ and $S=B^{L, 2}(t)$ in (2.11), then straightforward manipulations show that $B^{L, 2}$ solves the following integral equation

$$
B^{L, 2}(t)-K=C^{E}\left(B^{L, 2}(t), t\right)+\mathrm{E}_{t}\left[\int_{t}^{T_{2}} e^{-r(u-t)}\left(\delta S_{u}-r K\right) 1_{\left\{S_{u} \geq B^{L, 2}(u)\right\}} d u\right]
$$

on $\left[t^{*} \vee T_{1}, T_{2}\right]$ where $C^{E}$ is the price of the corresponding European uncapped call option. We note that this integral equation is exactly the same as the integral equation for the American uncapped call option boundary. This is not surprising as we know from Theorem 2.1 that $B^{L, 2}=B$ on $\left[t^{*} \vee T_{1}, T_{2}\right]$. Therefore, Theorem 2.3 provides alternative proof of this fact which was proven in Broadie and Detemple (1995).

In the next section we turn to the interval $\left[0, T_{1}\right]$.

\section{Main Result}

This section contains the main result of the paper, Theorem 3.1, which describes the optimal exercise policy on the interval $\left[0, T_{1}\right]$ for all cases of interest. The complete details, proofs and the option pricing formulas are given in Sections 4-6.

Theorem 3.1. (i) If $L_{1}<\min \left(L_{2}, B\left(T_{1}\right)\right)$, then the optimal exercise time in (2.3) is given by

$$
\tau^{*}(t)=\inf \left\{u \geq t:\left(S_{u}, u\right) \in \mathcal{E}\right\} \wedge T_{2}
$$

for $t \in\left[0, T_{1}\right)$, where the exercise region $\mathcal{E}=\mathcal{E}_{1} \cup \mathcal{E}_{2}$ consists of two disjoint regions

$$
\begin{aligned}
& \mathcal{E}_{1}=\left\{(S, t) \in \mathbb{R}^{+} \times\left[0, t^{1}\right]: L_{1} \leq S \leq B^{L, 1}(t)\right\} \\
& \mathcal{E}_{2}=\left\{(S, t) \in \mathbb{R}^{+} \times\left[T_{1}, T_{2}\right]: S \geq B^{L, 2}(t)\right\}
\end{aligned}
$$

for some $t^{1} \in\left[0, T_{1}\right)$ (specified in the next section). The exercise boundary $B^{L_{1}}=\left(B^{L, 1}(t)\right)_{t \in\left[0, t^{1}\right]}$ is characterized as

$$
B^{L, 1}(t)=L_{1} \quad \text { for } t \in\left[T_{0}, t^{1}\right]
$$

where $0 \leq T_{0} \leq t^{1}$ is described in the next section as well, and on the interval $\left[0, T_{0}\right]$ the boundary $B^{L, 1}$ solves the integral equation

$$
L_{1}-K=C^{E, L}\left(B^{L, 1}(t), t\right)+\Pi\left(B^{L, 1}(t), t ; B^{L, 1}(\cdot)\right)
$$

subject to the boundary condition $B^{L, 1}\left(T_{0}-\right)=L_{1}$, where the functions $C^{E, L}(S, t)$ and $\Pi\left(S, t ; B^{L, 1}(\cdot)\right)$ are given in (4.23) and (4.24), respectively. Moreover, if

$$
t^{0} \equiv T_{1}-\frac{1}{r} \log \left(\left(L_{2}-K\right) /\left(L_{1}-K\right)\right) \geq 0
$$


then $B^{L, 1}(t)=+\infty$ for $t \in\left[0, t^{0}\right]$ and $B^{L, 1}(t)<+\infty$ for $t \in\left(t^{0}, t^{1}\right]$.

(ii) If $B\left(T_{1}\right) \leq L_{1}<L_{2}$, then the optimal exercise time in (2.3) is given by

$$
\tau^{*}(t)=\inf \left\{u \geq t:\left(S_{u}, u\right) \in \mathcal{E}\right\} \wedge T_{2}
$$

for $t \in\left[0, T_{1}\right)$, where the exercise region $\mathcal{E}=\mathcal{E}_{1} \cup \mathcal{E}_{2}$ consists of

$$
\begin{aligned}
\mathcal{E}_{1}= & \left\{(S, t) \in \mathbb{R}^{+} \times\left[0, T_{1}\right): L_{1} \leq S \leq B^{L, 1}(t)\right\} \\
& \cup\left\{(S, t) \in \mathbb{R}^{+} \times\left[t^{*}, T_{1}\right): B(t) \leq S \leq L_{1}\right\} \\
\mathcal{E}_{2}= & \left\{(S, t) \in \mathbb{R}^{+} \times\left[T_{1}, T_{2}\right]: S \geq B(t)\right\} .
\end{aligned}
$$

The exercise boundary $B^{L_{1}}=\left(B^{L, 1}(t)\right)_{t \in\left[0, T_{1}\right)}$ is characterized as

$$
B^{L, 1}(t)=L_{1} \quad \text { for } t \in\left[T_{0}, T_{1}\right]
$$

where $0 \leq T_{0}<T_{1}$ (specified in Section 5), and on the interval $\left[0, T_{0}\right]$ the boundary $B^{L, 1}$ solves the integral equation

$$
L_{1}-K=C^{E, L}\left(B^{L, 1}(t), t\right)+\Pi\left(B^{L, 1}(t), t ; B^{L, 1}(\cdot)\right)
$$

subject to the boundary condition $B^{L, 1}\left(T_{0}-\right)=L_{1}$, where $C^{E, L}(S, t)$ and $\Pi\left(S, t ; B^{L, 1}(\cdot)\right)$ are given in (5.12) and (5.13), respectively. Moreover, if $t^{0} \equiv T_{1}-\frac{1}{r} \log \left(\left(L_{2}-K\right) /\left(L_{1}-K\right)\right) \geq 0$, then $B^{L, 1}(t)=+\infty$ for $t \in\left[0, t^{0}\right]$ and $B^{L, 1}(t)<+\infty$ for $t \in\left(t^{0}, T_{1}\right]$.

(iii) If $L_{1}>L_{2}$ and the cap is left-continuous, then the optimal exercise time in (2.3) is given by

$$
\tau^{*}(t)=\inf \left\{u \geq t:\left(S_{u}, u\right) \in \mathcal{E}\right\} \wedge T_{2}
$$

for $t \in\left[0, T_{1}\right)$, where the exercise region is

$$
\mathcal{E}=\left\{(S, t) \in \mathbb{R}^{+} \times\left[0, T_{1}\right]: S \geq B^{L, 1}(t)\right\} \cup\left\{(S, t) \in \mathbb{R}^{+} \times\left(T_{1}, T_{2}\right]: S \geq B(t) \wedge L_{2}\right\} .
$$

The optimal exercise boundary $B^{L, 1}=\left(B^{L, 1}(t)\right)_{t \in\left[0, T_{1}\right]}$ is decreasing and continuous on $\left[0, T_{1}\right)$ and is characterized as the solution to the integral equation

$$
B^{L, 1}(t) \wedge L_{1}-K=C^{E, L}\left(B^{L, 1}(t), t\right)+\Pi\left(B^{L, 1}(t), t ; B^{L, 1}(\cdot)\right)
$$

for $t \in\left[0, T_{1}\right)$ subject to the boundary condition $B^{L, 1}\left(T_{1}-\right)=\max \left(r K / \delta, L_{2} \wedge B\left(T_{1}\right)\right)$, where the functions $C^{E, L}(S, t)$ and $\Pi\left(S, t ; B^{L, 1}(\cdot)\right)$ are given in (6.10) and (6.11), respectively. The value of the boundary at $T_{1}$ is $B^{L, 1}\left(T_{1}\right)=L_{2} \wedge B\left(T_{1}\right)$.

Theorem 3.1 shows that the structure of the optimal exercise region over $\left[0, T_{1}\right]$ depends on the relative positions of the cap components $L_{1}, L_{2}$ and of the boundary $B\left(T_{1}\right)$ of the uncapped option at $T_{1}$. The first case is when $L_{1}<L_{2} \wedge B\left(T_{1}\right)$. In this instance, the exercise region involves the times $0 \leq t^{0} \vee 0 \leq T_{0} \leq t^{1}<T_{1}$. In the interval $\left(t^{1}, T_{1}\right)$, immediate exercise is always suboptimal: $(S, t) \notin \mathcal{E}$ for all $S \in \mathbb{R}^{+}$and $t \in\left(t^{1}, T_{1}\right)$. In $\left[T_{0}, t^{1}\right]$, immediate exercise is optimal along the line $S=L_{1}$ : for $t \in\left[T_{0}, t^{1}\right],(S, t) \in \mathcal{E}_{1}$ if and 
only if $S=L_{1}$. In $\left(t^{0}, T_{0}\right)$, it is optimal in the region between the boundaries $L_{1}$ and $B^{L, 1}=\left(B^{L, 1}(t)\right)_{t \in\left(t^{0}, T_{0}\right)}:$ for $t \in\left(t^{0}, T_{0}\right),(S, t) \in \mathcal{E}_{1}$ if and only if $L_{1} \leq S \leq B^{L, 1}(t)$. Finally, in $\left[0, t^{0}\right)$, it is optimal to exercise immediately above the cap $L_{1}$ : for $t \in\left[0, t^{0}\right)$, $(S, t) \in \mathcal{E}_{1}$ if and only if $S \geq L_{1}$. The most noteworthy aspect of the exercise region is that it splits into two parts $\mathcal{E}_{1}$ and $\mathcal{E}_{2}$, separated by a region, $\left\{(S, t): S \in \mathbb{R}^{+}, t \in\left(t^{1}, T_{1}\right)\right\}$, in which it is optimal to continue. Figure 6 displays the exercise region $\mathcal{E}=\mathcal{E}_{1} \cup \mathcal{E}_{2}$ and the times $\left(t^{0}, T_{0}, t^{1}\right)$ for this case.

The second case is when $B\left(T_{1}\right) \leq L_{1}<L_{2}$. In this instance $t^{1}=T_{1}$ and the exercise region involves the times $0 \leq t^{0} \vee 0 \leq T_{0}<T_{1}$. Thus, the continuation subregion separating the sets $\mathcal{E}_{1}$ and $\mathcal{E}_{2}$ has disappeared. But the exercise region $\mathcal{E}_{1}$ now includes the set $\left\{(S, t) \in \mathbb{R}^{+} \times\left[t^{*}, T_{1}\right): B(t) \leq S \leq L_{1}\right\}$ below the cap $L_{1}$. Figure 9 illustrates this structure of $\mathcal{E}=\mathcal{E}_{1} \cup \mathcal{E}_{2}$.

The last case is when $L_{1}>L_{2}$ and the cap is left-continuous, i.e., at time $T_{1}$ the cap is $L\left(T_{1}\right)=L_{1}$. In this instance immediate exercise is always optimal above the cap $L_{1}$ for $t \in\left[0, T_{1}\right]$. Moreover, there exists a lower boundary $B^{L}=\left(B^{L}(t)\right)_{t \in\left[0, T_{1}\right]}$ such that it is optimal to exercise at $(S, t)$ if $B^{L}(t) \leq S \leq L_{1}$ and $t \in\left[0, T_{1}\right]$. Figure 10 shows $\mathcal{E}$ for this case.

Intuition for the various components of the exercise region can be provided as follows. The time $t^{0}$, which appears in cases (i) and (ii), is the unique solution to the equation $e^{-r\left(T_{1}-t^{0}\right)}\left(L_{2}-K\right)=L_{1}-K$. If $t^{0} \geq 0$, then the left-hand side of this equation is the value at $t^{0} \in\left[0, T_{1}\right]$ from receiving the maximum payoff on the contract at the earliest possible date, $T_{1}$. At times $t$ prior to $t^{0}$, immediate exercise above the cap $L_{1}$ pays off $L_{1}-K>e^{-r\left(T_{1}-t\right)}\left(L_{2}-K\right)$, hence cannot be dominated by any waiting policy.

The time $t^{1}$, which plays a major role in case (i), is determined by the policy of waiting until the jump time $T_{1}$ before deciding to exercise or not. The value of this particular waiting policy is the value $C^{w}\left(S, t^{1}\right)$ of a European compound option with maturity date $T_{1}$ paying the American capped call option price at time $T_{1}$. As $L_{1}<\min \left(L_{2}, B\left(T_{1}\right)\right)$, the payoff at $\left(L_{1}, T_{1}\right)$ is $C^{A, L_{2}}\left(L_{1}, T_{1}\right)>L_{1}-K$. Hence, immediate exercise is suboptimal at the point $\left(L_{1}, T_{1}\right)$. Moving back in time along the cap $L_{1}$, continuously changes the value $C^{w}\left(L_{1}, t\right)$ of the compound contract, because of discounting and uncertainty in the underlying asset price, but the immediate exercise payoff remains the same. The time $t^{1}$ is the largest time in $\left[t^{0}, T_{1}\right)$ at which $C^{w}\left(L_{1}, t\right)=L_{1}-K$. For any $t \in\left(t^{1}, T_{1}\right), C^{w}\left(L_{1}, t\right)>L_{1}-K$ and immediate exercise at $\left(L_{1}, t\right)$ is dominated. Moreover, because $C^{w}(S, t)$ is an increasing function of $S, C^{w}(S, t)>L_{1}-K$ and immediate exercise at $(S, t)$ is also dominated for $S>L_{1}$ and $t \in\left(t^{1}, T_{1}\right)$. That immediate exercise is dominated for $S<L_{1}$ and $t \in\left(t^{1}, T_{1}\right)$ follows from the fact that a capped option with cap $L_{1}$ and maturity date $T_{1}$ would not be exercised at this point.

Time $t^{1}$ is the largest time in $\left[t^{0}, T_{1}\right)$ at which it becomes optimal to exercise along the cap. As $C^{w}\left(\cdot, t^{t}\right)$ is strictly increasing and the exercise payoff of the capped option does not change above the cap, $C^{w}\left(S, t^{1}\right)>L_{1}-K$ for $S>L_{1}$ and immediate exercise remains suboptimal at $\left(S, t^{1}\right)$ for $S>L_{1}$. It is also suboptimal at $\left(S, t^{1}\right)$ for $S<L_{1}$ for the reasons indicated above. This knife-edge property at $S=L_{1}$ persists as one moves further back in time along the cap. The time $T_{0}$ marks the end of that stretch. For $t<T_{0}$, immediate exercise can become optimal above the cap, specifically for $L_{1} \leq S \leq B^{L, 1}(t)$ where $B^{L, 1}=\left(B^{L, 1}(t)\right)_{t \in\left(t^{0}, T_{0}\right)}$ is an endogenous boundary. 
Intuition for case (iii) is as follows. As we impose left-continuity of the cap, we know the optimal exercise strategy on $\left(T_{1}, T_{2}\right]$. At time $t=T_{1}$, we compare the immediate payoff $S \wedge L_{1}-K$ with the continuation value, i.e., the price of the capped option $C^{A, L_{2}}\left(S, T_{1}\right)$ (with cap $L_{2}$ ). Clearly, immediate exercise is optimal if and only if $S$ is above the critical threshold $b^{L, 1}\left(T_{1}\right)=L_{2} \wedge B\left(T_{1}\right)$. It then remains to understand the exercise policy on $\left[0, T_{1}\right)$. Immediate exercise above $L_{1}$ is always optimal because $L_{1}-K$ is the maximum payoff that can be attained. As the cap is non-increasing in this case, we have that there exists a boundary $B^{L, 1}$ on $\left[0, T_{1}\right)$ below the cap $L_{1}$ such that it is optimal to exercise if $B^{L, 1}(t) \leq S \leq L_{1}$. Furthermore, one should not exercise if $S<r K / \delta \wedge L_{1}$, because the local benefits of waiting to exercise are positive in this region. Near $T_{1}$, it is optimal to exercise the option when $S \geq \max \left(r K / \delta, L_{2} \wedge B\left(T_{1}\right)\right)$ as these benefits are negative. Therefore, there is a part of the exercise region below $L_{1}$ and prior to $T_{1}$ if and only if $r K / \delta<L_{1}$.

Remark 3.2. (Non dividend-paying asset) If $\delta=0$, then it is never optimal to exercise the American uncapped call option early, i.e., $B=+\infty$ on $\left[0, T_{2}\right]$. This immediately excludes the second case. The exercise region on $\left[T_{1}, T_{2}\right]$ is simply the set above $L_{2}$. The structure of $\mathcal{E}_{1}$ on $\left[0, T_{1}\right)$, in the first and third cases, has the form described above. The condition $\delta=0$ does not simplify it. To summarize, the only qualitative change in this case is that the exercise boundary on $\left[T_{1}, T_{2}\right]$ becomes the constant cap $L_{2}$.

Remark 3.3. (Constant cap) If $L_{1}=L_{2}$, then the contract simply becomes the American capped option with single cap $L=L_{1}=L_{2}$ from Broadie and Detemple (1995) (see the results of Section 2).

\section{Case $L_{1}<\min \left(L_{2}, B\left(T_{1}\right)\right)$}

In this section we assume that $L_{1}<B\left(T_{1}\right)$ or, equivalently, that $t^{*} \geq T_{1}$, and the goal is to establish the statements of Theorem $3.1(i)$ above. The complete analysis of this case is divided into steps 1-8 below.

Proof of Theorem 3.1 $(i)$. 1. Let us define the function

$$
C^{w}(S, t) \equiv \mathrm{E}_{t}\left[e^{-r\left(T_{1}-t\right)} C^{A, L_{2}}\left(S_{T_{1}}, T_{1}\right)\right]=\mathrm{E}_{t}\left[e^{-r\left(\tau^{*}\left(T_{1}\right)-t\right)}\left(S_{\tau^{*}\left(T_{1}\right)} \wedge L_{2}-K\right)^{+}\right]
$$

for $t<T_{1}$ and $S>0$, where $\tau^{*}\left(T_{1}\right)$ is defined in (2.6) and in the second equality we used Theorem 2.1. The function $C^{w}(S, t)$ is the value of a European derivative with maturity date $T_{1}$ and payoff given by the American capped call option price with cap $L_{2}$. It can also be viewed as an American capped call option with delayed exercise provision restricted to $\left[T_{1}, T_{2}\right]$. For the holder of the original capped option (2.3), $C^{w}(S, t)$ is the value of the policy which consists in waiting until at least $T_{1}$ before deciding to exercise or not. This waiting policy is always feasible and its value provides a lower bound for $C^{A, L}$. By using the Ito-Tanaka formula for $C^{A, L_{2}}$ we can rewrite $C^{w}$ as

$$
\begin{aligned}
C^{w}(S, t) & =\mathrm{E}_{t}\left[e^{-r\left(T_{1}-t\right)} C^{A, L_{2}}\left(S_{T_{1}}, T_{1}\right)\right] \\
& =C^{A, L_{2}}(S, t)-r\left(L_{2}-K\right) \mathrm{E}_{t}\left[\int_{t}^{T_{1}} e^{-r(u-t)} 1_{\left.\left\{S_{u} \geq L_{2}\right)\right\}} d u\right]
\end{aligned}
$$




$$
\begin{aligned}
& -\frac{1}{2} \mathrm{E}_{t}\left[\int_{t}^{T_{1}} e^{-r(u-t)} C_{S}^{A, L_{2}}\left(L_{2}-, u\right) d \ell_{u}^{L_{2}}\right] \\
= & C^{E, L_{2}}(S, t)+r\left(L_{2}-K\right) \mathrm{E}_{t}\left[\int_{T_{1}}^{T_{2}} e^{-r(u-t)} 1_{\left.\left\{S_{u} \geq L_{2}\right)\right\}} d u\right] \\
& +\mathrm{E}_{t}\left[\int_{t}^{T_{2}} e^{-r(u-t)}\left(\delta S_{u}-r K\right) 1_{\left\{B(u) \leq S_{u} \leq L_{2}\right\}} d u\right] \\
& +\frac{1}{2} \mathrm{E}_{t}\left[\int_{T_{1}}^{T_{2}} e^{-r(u-t)} C_{S}^{A, L_{2}}\left(L_{2}-, u\right) d \ell_{u}^{L_{2}}\right]
\end{aligned}
$$

for $S>0$ and $t<T_{1}$. We then use (2.14) to calculate $d \mathrm{E}_{t}\left[\ell_{u}^{L_{2}}\right]$, the derivative $C_{S}^{A, L_{2}}\left(L_{2}-, u\right)$ is estimated using (2.9), and $\mathrm{E}_{t}\left[1_{\left\{S_{u} \geq L_{2}\right\}}\right], \mathrm{E}_{t}\left[1_{\left\{B(u) \leq S_{u} \leq L_{2}\right\}}\right], \mathrm{E}_{t}\left[S_{u} 1_{\left\{B(u) \leq S_{u} \leq L_{2}\right\}}\right]$ can be written in terms of the cumulative distribution function $\Phi(\cdot)$ of the standard normal law.

As $L_{1}<B\left(T_{1}\right)$, we have that $C^{w}\left(L_{1}, T_{1}\right)>L_{1}-K$. Then the unique solution $B^{w}\left(T_{1}\right)$ to

$$
C^{w}\left(B^{w}\left(T_{1}\right), T_{1}\right)=L_{1}-K
$$

is such that $B^{w}\left(T_{1}\right)<L_{1}$. Similarly, for $t \in\left[0, T_{1}\right)$, let $B^{w}(t)$ be the unique solution to

$$
C^{w}\left(B^{w}(t), t\right)=L_{1}-K
$$

and note that

$$
\begin{cases}C^{w}(S, t)>L_{1}-K & \text { for } S>B^{w}(t) \\ C^{w}(S, t)<L_{1}-K & \text { for } S<B^{w}(t)\end{cases}
$$

The boundary $B^{w}=\left(B^{w}(t)\right)_{t \in\left[0, T_{1}\right]}$ represents the continuous curve at which the waiting policy associated with $C^{w}$ described above, has the same value as $L_{1}-K$, that is the largest possible payoff on $\left[0, T_{1}\right)$. The following result is an obvious consequence.

Lemma 4.1. Immediate exercise of the capped option is suboptimal if $t \in\left[0, T_{1}\right]$ and $S>$ $B^{w}(t)$ or $S<L_{1}$.

Proof. If $S>B^{w}(t)$, then $C^{w}(S, t)>L_{1}-K \geq S \wedge L_{1}-K$ implying that immediate exercise is strictly dominated by the policy of waiting until time $T_{1}$ before considering exercise. If $S<$ $L_{1}$, then there exists an American uncapped call option $C^{A}\left(S, t ; T_{0}\right)$ with shorter maturity $T_{0} \leq T_{1}$ and corresponding optimal exercise boundary $B^{0}$ such that $S<B^{0}(t)<L_{1}$ and thus $C^{A}\left(S, t ; T_{0}\right)>S-K$. As the policy $B^{0}$ is feasible for the capped option holder and the payoff matches the payoff of the uncapped option, it follows that $C^{A, L}(S, t) \geq C^{A}\left(S, t ; T_{0}\right)>S-K$. Immediate exercise is therefore suboptimal for the original American capped option.

2. Let us define $t^{1}$ as the largest root of $B^{w}(t)=L_{1}$ on $\left[0, T_{1}\right]$ if one exists, otherwise we let $t^{1}=0$, and note that $t^{1}<T_{1}$ as $B^{w}\left(T_{1}\right)<L_{1}$. Also define the set

$$
\mathcal{D}^{w}=\left\{(S, t) \in \mathbb{R}^{+} \times\left[0, t^{1}\right]: L_{1} \leq S \leq \max \left(B^{w}(t), L_{1}\right)\right\}
$$

which represents the region with lower boundary $L_{1}$ and upper boundary $\max \left(B^{w}, L_{1}\right)$ on $\left[0, t^{1}\right]$ (see Figure 4 for illustration). Now we are ready to provide further insights into the structure of the optimal exercise region. 


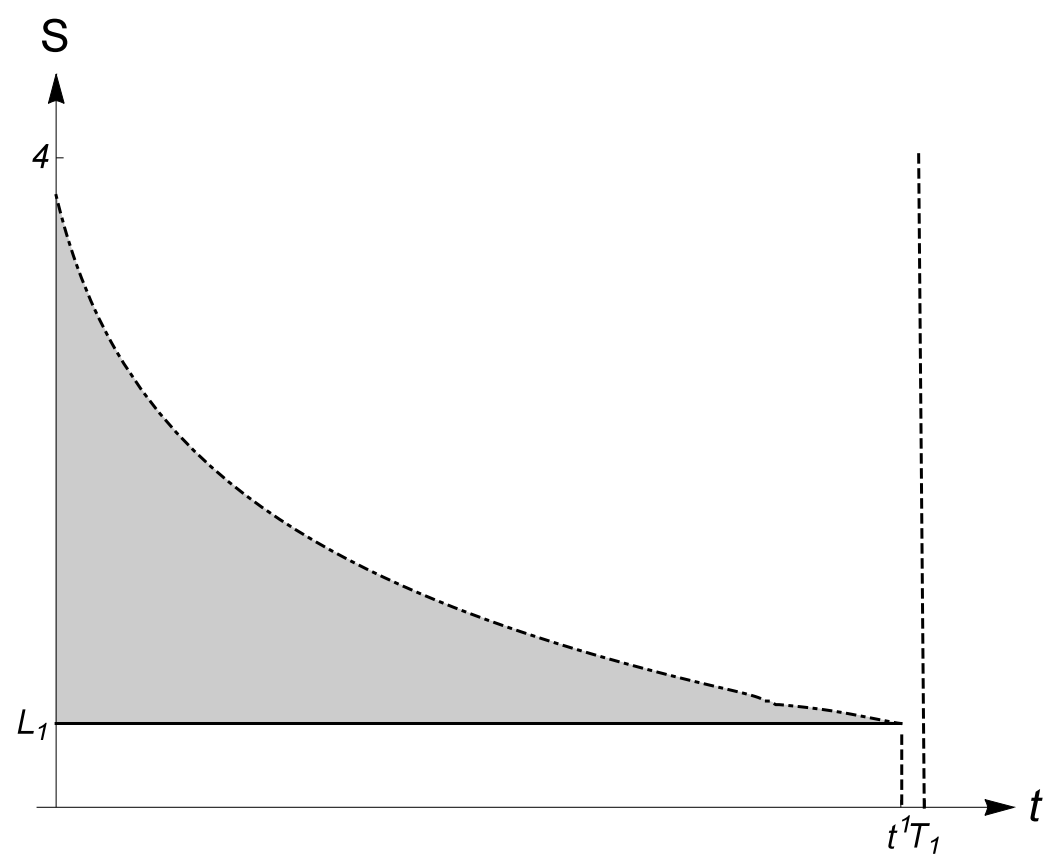

Figure 4. This figure plots the region $\mathcal{D}^{w}$ (gray). The dot-dashed line represents the upper boundary $B^{w}$. The parameter set is $T_{1}=3, T_{2}=4, K=1, L_{1}=$ $1.3, L_{2}=1.39, r=0.1, \delta=0.1, \sigma=0.3$. The value of $t^{1}$ is 2.93 .

Theorem 4.2. ( $i$ ) The immediate exercise region of the capped call option (2.3) consists of two disconnected subregions $\mathcal{E}=\mathcal{E}_{1} \cup \mathcal{E}_{2}$ where $\mathcal{E}_{1} \subseteq \mathcal{D}^{w}$ and

$$
\mathcal{E}_{2}=\left\{(S, t) \in \mathbb{R}^{+} \times\left[T_{1}, T_{2}\right]: S \geq B^{L, 2}(t)\right\} .
$$

(ii) The subregion $\mathcal{E}_{1}$ is down-connected above $L_{1}$ and non-empty if $t^{1}>0$. If $t^{1}=0$ and $B^{w}(0)=L_{1}$, it consists of the single point $\left(L_{1}, t^{1}\right)$. If $t^{1}=0$ and $B^{w}(0)<L_{1}$, then $\mathcal{E}_{1}=\varnothing$.

(iii) $\left(L_{1}, v\right) \in \mathcal{E}_{1}$ for all $v \in\left[0, t^{1}\right]$.

(iv) If $t^{0} \equiv T_{1}-\frac{1}{r} \log \left(\left(L_{2}-K\right) /\left(L_{1}-K\right)\right) \geq 0$, then $(S, t) \in \mathcal{E}_{1}$ for any $t \in\left[0, t^{0}\right]$ and $S \geq L_{1}$. Also, for any $t \in\left(t_{0}, T_{1}\right)$ there exists $\widetilde{s}=\widetilde{s}(t)$ such that for any $S>\widetilde{s}$ it is optimal to continue at $(S, t)$.

$(v)$ There exists $T_{0} \in\left[0, t^{1}\right]$ such that it is not optimal to exercise at $(S, t)$ for $S>L_{1}$ and $t \in\left[T_{0}, t^{1}\right]$.

Proof. (i) By Lemma 4.1, immediate exercise is suboptimal above $B^{w}$ and below $L_{1}$. It follows immediately that $\mathcal{E}_{1} \subseteq \mathcal{D}^{w}$.

(ii) To show that $\mathcal{E}_{1}$ is down-connected above $L_{1}$, suppose that $(S, t) \in \mathcal{E}_{1}$ and consider a point $\left(S^{\prime}, t\right)$ such that $S \geq S^{\prime} \geq L_{1}$. As $S$. $\geq S^{\prime}$. it follows that $C^{A, L}(S, t) \geq C^{A, L}\left(S^{\prime}, t\right)$. Optimality of immediate exercise at $(S, t)$ implies $L_{1}-K=C^{A, L}(S, t) \geq C^{A, L}\left(S^{\prime}, t\right) \geq$ $L_{1}-K$. Thus $\left(S^{\prime}, t\right) \in \mathcal{E}_{1}$, which establishes down-connectedness. To show non-emptiness, let $t^{1}>0$ and note that $C^{w}\left(L_{1}, t^{1}\right)=L_{1}-K$. In $\left(t^{1}, T_{1}\right]$, immediate exercise is suboptimal, so the value of the American capped option is the same as the value of the option with exercise 
delayed to the interval $\left[T_{1}, T_{2}\right]$, i.e., $C^{A, L}(S, t)=C^{w}(S, t)$. If $t=t^{1}$ and $S=L_{1}$, then there are only two choices, exercise or follow the delayed exercise policy. Both produce the same value. Thus, $C^{A, L}\left(L_{1}, t^{1}\right)=C^{w}\left(L_{1}, t^{1}\right)=L_{1}-K$ and $\left(L_{1}, t^{1}\right) \in \mathcal{E}_{1}$. The same argument applies if $t^{1}=0$ and $B^{w}(0)=L_{1}$. In this case, the region consists of a single point, $\mathcal{D}=\left\{\left(L_{1}, t^{1}\right)\right\}$ and $\mathcal{E}_{1}=\mathcal{D}$. Finally, if $t^{1}=0$ and $B^{w}(0)<L_{1}$, immediate exercise is suboptimal for all $S \in \mathbb{R}^{+}$, implying $\mathcal{E}_{1}=\varnothing$.

(iii) From $(i)$ we have that $\left(L_{1}, t^{1}\right) \in \mathcal{E}_{1}$ and let us take $\left(L_{1}, t\right)$ for any $t \in\left[0, t^{1}\right]$. Now, we introduce an auxiliary capped option $C^{A, \widetilde{L}}$ with earlier maturity $\widetilde{T}_{1}<T_{1}$ for the cap $L_{1}$ so that the new cap is given as $\widetilde{L}_{t}=L_{1} 1_{t<\widetilde{T}_{1}}+L_{2} 1_{\widetilde{T}_{1} \leq t \leq T_{2}}$. Clearly $C^{A, L} \leq C^{A, \widetilde{L}}$, then we can decrease $\widetilde{T}_{1}$ continuously such that the point $\widetilde{t}^{1}<\widetilde{T}_{1}$ (which is defined in the same way as $t^{1}$ for $\left.T_{1}\right)$ coincides with $t$. From $(i)$ we then have that $C^{A, \widetilde{L}}\left(L_{1}, \widetilde{t}^{1}\right)=L_{1}-K$ and thus $L_{1}-K \leq C^{A, L}\left(L_{1}, t\right) \leq C^{A, \widetilde{L}}\left(L_{1}, \widetilde{t}^{1}\right)=L_{1}-K$, i.e., $\left(L_{1}, t\right) \in \mathcal{E}_{1}$.

(iv) Now if we assume that $t^{0}=T_{1}-\frac{1}{r} \log \left(\left(L_{2}-K\right) /\left(L_{1}-K\right)\right) \geq 0$ and take $t \leq t^{0}$. Then for any exercise policy $\tau$ we have that

$$
\begin{aligned}
& \mathrm{E}_{t}\left[e^{-r(\tau-t)}\left(\left(L_{1} \wedge S_{\tau}-K\right) 1_{\left\{\tau<T_{1}\right\}}+\left(L_{2} \wedge S_{\tau}-K\right) 1_{\left\{\tau \geq T_{1}\right\}}\right)\right] \\
& \leq\left(L_{1}-K\right) Q_{t}\left(\tau<T_{1}\right)+e^{-r\left(T_{1}-t\right)}\left(L_{2}-K\right) Q_{t}\left(\tau \geq T_{1}\right) \leq L_{1}-K
\end{aligned}
$$

and therefore the immediate exercise payoff $L_{1}-K$ dominates the expected value of any admissible exercise strategy. Thus, it is optimal to exercise at once for any $t \leq t^{0}$ and $S \geq L_{1}$.

Now, we take any $t \in\left(t^{0}, T_{1}\right)$. Then choose large enough $\widetilde{s}=\widetilde{s}(t)>0$ such that the probability $e^{r\left(T_{1}-t\right)}\left(L_{1}-K\right) /\left(L_{2}-K\right) \leq Q_{t}\left(S_{T_{1}}>L_{2}\right)<1$. If we consider the strategy of exercising at $T_{1}$, then clearly

$$
C^{A, L}(S, t) \geq \mathrm{E}_{t}\left[e^{-r\left(T_{1}-t\right)}\left(L_{2}-K\right) 1_{\left\{S_{T_{1}}>L_{2}\right\}}\right]>\left(L_{2}-K\right) e^{-r\left(T_{1}-t\right)} Q_{t}\left(S_{T_{1}}>L_{2}\right)>L_{1}-K
$$

for $S>\widetilde{s}$ as $t>t^{0}$ and hence it is not optimal to stop at $(S, t)$. Thus, we proved that there exists $\widetilde{s}$ such that for any $S>\widetilde{s}$ it is optimal to continue at $(S, t)$.

$(v)$ We know the optimal exercise strategy on $\left[t^{1}, T_{1}\right]$ and proved above that points $\left(L_{1}, t\right) \in \mathcal{E}_{1}$ for $t \in\left[0, t^{1}\right]$. Now let us consider the points $(S, t)$, where $S>L_{1}$ and $t<t^{1}$, and the exercise strategy given by the first hitting time of the cap $L_{1}$ if it occurs before $t_{1}$, otherwise we follow the already known optimal exercise rule starting from $t^{1}$. The value of this strategy can be computed as

$$
C^{0}(S, t)=\left(L_{1}-K\right) \mathrm{E}_{t}\left[e^{-r\left(\tau_{L_{1}}-t\right)} 1_{\left\{\tau_{L_{1}}<t^{1}\right\}}\right]+\mathrm{E}_{t}\left[e^{-r\left(t^{1}-t\right)} C^{w}\left(S_{t^{1}}, t^{1}\right) 1_{\left\{\tau_{L_{1}} \geq t^{1}\right\}}\right]
$$

where $\tau_{L_{1}}=\inf \left\{u \geq t: S_{u}=L_{1}\right\}$ denotes the first hitting time of $L_{1}$. We refer to Lemma 8.1 in Appendix in order to compute both expectations in (4.7). In general, we have that there exists $T_{0} \in\left[0, t^{1}\right]$ (numerical results show that $T_{0}<t^{1}$, see Figure 5 ) such that $C^{0}(S, t)>L_{1}-K$ for $S>L_{1}$ and $t \in\left[T_{0}, t^{1}\right]$. Therefore, it is optimal not to exercise and $C^{A, L}=C^{0}$ for $S>L_{1}$ and $t \in\left[T_{0}, t^{1}\right]$.

3. From Theorem 3.2, we have that $C^{A, L}=C^{w}$ on $\left\{(S, t) \in \mathbb{R}^{+} \times\left[t^{1}, T_{1}\right]\right\}$ and it is natural to introduce the upper exercise boundary of $\mathcal{E}_{1}$ in the case $t^{1}>0$. 


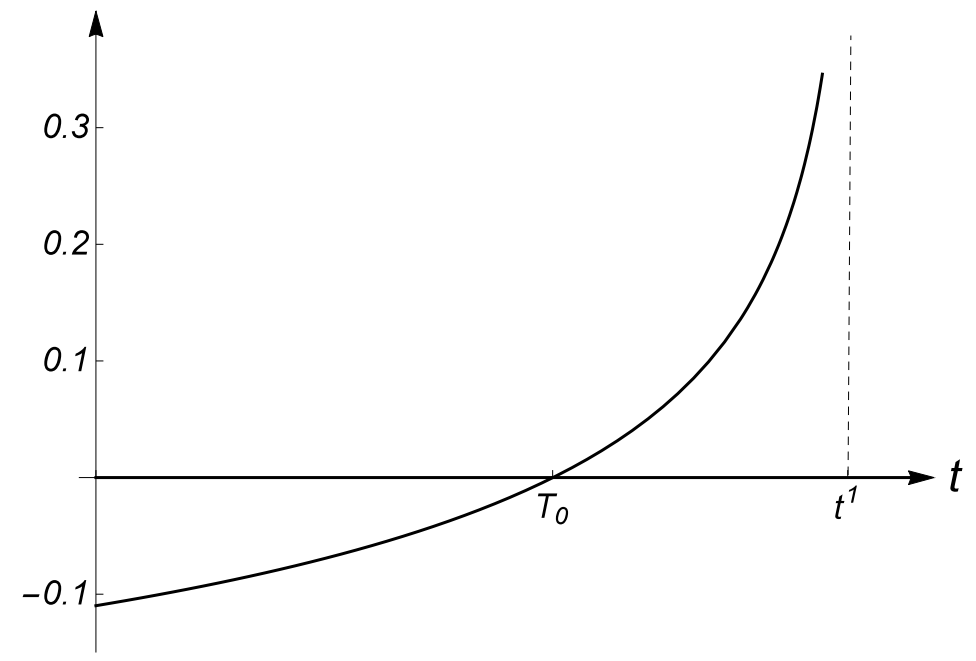

Figure 5. This figure plots the right-hand side derivative $C_{S}^{0}\left(L_{1}+, t\right)$ of $C^{0}$ at $L_{1}$ for $t \in\left[0, t^{1}\right)$. The parameter set is $T_{1}=3, T_{2}=4, K=1, L_{1}=1.3, L_{2}=$ $1.39, r=0.1, \delta=0.1, \sigma=0.3$. We obtain $T_{0}=1.78$.

Definition 4.3. We define the upper exercise boundary $B^{L, 1}=\left(B^{L, 1}(t)\right)_{t \in\left[0, t^{1}\right]}$ such that the immediate exercise subregion $\mathcal{E}_{1}$ can be written as

$$
\mathcal{E}_{1}=\left\{(S, t) \in \mathbb{R}^{+} \times\left[0, t^{1}\right]: L_{1} \leq S \leq B^{L, 1}(t)\right\}
$$

From Theorem 3.2, we immediately have the following properties of $B^{L, 1}$ : a) $B^{L, 1}(t) \leq$ $B^{w}(t)$ for $t \in\left[0, t^{1}\right]$; b) $B^{L, 1}(t)=L_{1}$ for $t \in\left[T_{0}, t^{1}\right]$; c) $B^{L, 1}(t)<+\infty$ for $t>t^{0} \vee 0$ and if $t^{0} \geq 0$ then $B^{L, 1}(t)=+\infty$ for $t \in\left[0, t^{0}\right]$.

Using the property b) above, we can rewrite $\mathcal{E}_{1}$ as $\widetilde{\mathcal{E}}_{1} \cup \overline{\mathcal{E}}_{1}$ where

$$
\begin{aligned}
& \widetilde{\mathcal{E}}_{1}=\left\{(S, t) \in \mathbb{R}^{+} \times\left[0, T_{0}\right]: L_{1} \leq S \leq B^{L, 1}(t)\right\} \\
& \overline{\mathcal{E}}_{1}=\left\{(S, t) \in \mathbb{R}^{+} \times\left[T_{0}, t^{1}\right]: S=L_{1}\right\}
\end{aligned}
$$

4. Next, we provide lower and upper bounds for $\mathcal{E}_{1}$ using simple benchmarks. First, let us consider the capped option price $C^{A, L, \infty}$ with infinite maturity $T_{2}$ and cap $L_{\tau}^{\infty}=$ $L_{1} 1_{\tau<T_{1}}+L_{2} 1_{T_{1} \leq \tau<\infty}$. Let $\mathcal{E}^{\infty}$ be the immediate exercise region for this option and define the boundary $B^{w, \infty}=\left(B^{w, \infty}(t)\right)_{t \in\left[0, T_{1}\right]}$, time $t^{1, \infty}$ and region $\mathcal{D}^{w, \infty}$ in the same way as $B^{w}$, $t^{1}$ and $\mathcal{D}^{w}$, respectively. Clearly, $\mathcal{E}^{\infty}=\mathcal{E}_{1}^{\infty} \cup \mathcal{E}_{2}^{\infty}$ where $\mathcal{E}_{1}^{\infty} \subseteq \mathcal{D}^{w, \infty}$ satisfies properties (ii) - $(v)$ in Theorem 4.2 and $\mathcal{E}_{2}^{\infty}=\left\{(S, t) \in \mathbb{R}^{+} \times\left[T_{1}, T_{2}\right): S \geq B^{\infty} \wedge L_{2}\right\}$, where $B^{\infty}$ is the optimal threshold of the uncapped perpetual call option. Moreover, the following lemma holds.

Lemma 4.4. The exercise subregion $\mathcal{E}_{1}^{\infty}$ is left-connected: if $(S, t) \in \mathcal{E}_{1}^{\infty}$ for $t \leq t^{1, \infty}$ and $S>L_{1}$, then $(S, v) \in \mathcal{E}_{1}^{\infty}$ for all $v \in[0, t]$.

Proof. The proof is by contradiction. Suppose that $(S, t) \in \mathcal{E}_{1}^{\infty}$ for $t \leq t^{1, \infty}$ and $S>L_{1}$ and that there exists $v \in[0, t]$ such that $(S, v) \notin \mathcal{E}_{1}^{\infty}$, i.e., $C^{A, L, \infty}(S, v)>L_{1}-K$. Let $\tau_{v}^{\infty}$ 
be the optimal exercise policy at $(S, v)$. Now consider the translated capped contract with $L_{\tau}^{t}=L_{1} 1_{\tau<T_{1}+t-v}+L_{2} 1_{T_{1}+t-v \leq \tau<\infty}$ and price $C^{A, L, \infty}\left(S, s ; T_{1}+t-v\right)$ for $s \in[0, \infty)$. Note that $C^{A, L, \infty}\left(S, \cdot ; T_{1}+t-v\right) \leq C^{A, L, \infty}(S, \cdot)$. As $C^{A, L, \infty}\left(S, t ; T_{1}+t-v\right)=C^{A, L, \infty}(S, v)$, it then follows that $C^{A, L, \infty}(S, v) \leq C^{A, L, \infty}(S, t)$. Hence, given the initial assumptions, $L_{1}-K<$ $C^{A, L, \infty}(S, v) \leq C^{A, L, \infty}(S, t)=L_{1}-K$, we arrive at a contradiction. It therefore must be that $(S, v) \in \mathcal{E}_{1}^{\infty}$.

If we define the upper boundary $B^{L, 1, \infty}=\left(B^{L, 1, \infty}(t)\right)_{t \in\left[0, t^{1, \infty}\right]}$ of $\mathcal{E}_{1}^{\infty}$, then left-connectedness of $\mathcal{E}_{1}^{\infty}$ implies that the boundary $B^{L, 1, \infty}$ is non-increasing. Moreover, as $C^{A, L, \infty} \geq C^{A, L}$, we have that $\mathcal{E}_{1}^{\infty} \subseteq \mathcal{E}_{1}$ and hence the boundary $B^{L, 1, \infty}$ is a lower bound for $B^{L, 1}$.

Next, we consider the contract with restricted exercise provision, limited to $\left[0, T_{1}\right]$, and automatic exercise at $L_{2}$ in $\left[T_{1}, T_{2}\right]$. Let $C^{A, L, T_{1}}(S, v)$ be the contract price and $\mathcal{E}^{T_{1}}$ be the immediate exercise region. We define the boundary $B^{w, T_{1}}=\left(B^{w, T_{1}}(t)\right)_{t \in\left[0, T_{1}\right]}$ and region $\mathcal{D}^{w, T_{1}}$ in the same way as in (4.3) and (4.4), respectively. Note that $\mathcal{E}^{T_{1}}=\mathcal{E}_{1}^{T_{1}} \cup \mathcal{E}_{2}^{T_{1}}$ where $\mathcal{E}_{1}^{T_{1}} \subseteq$ $\mathcal{D}^{w, T_{1}}$ satisfies properties $(i i)$ - $(v)$ in Theorem 4.2 and $\mathcal{E}_{2}^{T_{1}}=\left\{(S, t) \in \mathbb{R}^{+} \times\left[T_{1}, T_{2}\right]: S \geq L_{2}\right\}$ is the region with automatic exercise at $L_{2}$. It is clear that $C^{A, L, T_{1}}(S, t) \leq C^{A, L}(S, t)$ for all $t \in\left[0, T_{2}\right]$, so that $\mathcal{E}_{1} \subseteq \mathcal{E}_{1}^{T_{1}}$.

To summarize, we have the following lemma.

Lemma 4.5. $\mathcal{E}_{1}^{\infty} \subseteq \mathcal{E}_{1} \subseteq \mathcal{E}_{1}^{T_{1}}$, where $\mathcal{E}_{1}^{\infty}$ and $\mathcal{E}_{1}^{T_{1}}$ satisfy the properties of Theorem 4.2.

5. We already know the optimal exercise strategy and the option price on interval $\left[T_{0}, T_{2}\right]$, so that we can set $T_{0}$ as the new maturity date of the contract. Thus, the American capped option (2.3) with discontinuous cap $L_{\tau}=L_{1} 1_{\left\{\tau<T_{1}\right\}}+L_{2} 1_{\left\{T_{1} \leq t \leq T_{2}\right\}}$ and maturity date $T_{2}$ is equivalent to an American capped derivative with maturity date $T_{0}$ and exercise payoff

$$
G\left(S_{\tau}, \tau\right)=\left(S_{\tau} \wedge L_{1}-K\right)^{+} 1_{\left\{\tau<T_{0}\right\}}+C^{0}\left(S_{\tau}, \tau\right) 1_{\left\{\tau=T_{0}\right\}}
$$

at $\tau \in\left[0, T_{0}\right]$. The equivalent contract has a constant cap $L_{1}$, in the interval $\left[0, T_{0}\right)$ and terminal value at $T_{0}$ given as the known value of the American capped option $C^{A, L}\left(S_{T_{0}}, T_{0}\right)=$ $C^{0}\left(S_{T_{0}}, T_{0}\right)$ (see (4.7) for the definition of $C^{0}$ ).

For this contract, the instantaneous benefits of waiting to exercise are given by

$$
H(S) \equiv\left(\mathbb{L}_{S} G-r G\right)(S)
$$

for $t<T_{0}$ and thus

$$
H(S)=h_{1}(S) 1_{\left\{K \leq S<L_{1}\right\}}+h_{2} 1_{\left\{S \geq L_{1}\right\}}
$$

for $S>0$ where

$$
h_{1}(S)=(r-\delta) S-r(S-K)=r K-\delta S \quad \text { and } \quad h_{2}=-r\left(L_{1}-K\right) .
$$

6. Standard Markovian arguments lead to the following free-boundary problem for the value function $C^{A, L}=C^{A, L}(S, t)$ and the upper exercise boundary $B^{L, 1}=\left(B^{L, 1}(t)\right)_{t \in\left[0, T_{0}\right]}$ to be determined

$$
C_{t}^{A, L}+\mathbb{L}_{S} C^{A, L}-r C^{A, L}=0 \quad \text { in } \mathcal{C}_{1}
$$




$$
\begin{array}{lr}
C^{A, L}\left(B^{L, 1}(t)+, t\right)=L_{1}-K & \text { for } t \in\left[0, T_{0}\right) \\
C^{A, L}(S, t)>G(S, t) & \text { in } \mathcal{C}_{1} \\
C^{A, L}(S, t)=G(S, t) & \text { in } \widetilde{\mathcal{E}}_{1}
\end{array}
$$

where the continuation set $\mathcal{C}_{1}$ and the exercise region $\widetilde{\mathcal{E}}_{1}$ on $\left[0, T_{0}\right)$ are given by

$$
\begin{aligned}
& \mathcal{C}_{1}=\left\{(S, t) \in \mathbb{R}^{+} \times\left[0, T_{0}\right]: S<L_{1} \text { or } S>B^{L, 1}(t)\right\} \\
& \widetilde{\mathcal{E}}_{1}=\left\{(S, t) \in \mathbb{R}^{+} \times\left[0, T_{0}\right]: L_{1} \leq S \leq B^{L, 1}(t)\right\}
\end{aligned}
$$

Remark 4.6. We note that the system above does not impose a smooth-fit condition at $B^{L, 1}$. In typical stopping time problems this property follows from the fact that an underlying process immediately enters into the exercise region if it starts just above or below the exercise boundary. However, in our problem, the boundary $B^{L, 1}$ is not always increasing. Therefore, this standard argument cannot be applied and we are not able to prove the smooth-fit property. Intuitively, it should hold and the numerical results support this intuition. But in order to keep the analysis as rigorous as possible, we do not assume that the smooth-fit condition holds and thus a local time term will appear in the pricing formulas.

7. We now assume that the technical conditions required on $B^{L, 1}$ for the local time-space formula on curves (Peskir (2005)) are satisfied, i.e., $B^{L, 1}$ is continuous and of bounded variation on $\left[0, T_{0}\right)$. We then apply the formula to $e^{-r\left(T_{0}-t\right)} C^{A, L}\left(S_{T_{0}}, T_{0}\right)$ and obtain

$$
\begin{aligned}
& e^{-r\left(T_{0}-t\right)} C^{A, L}\left(S_{T_{0}}, T_{0}\right) \\
& =C^{A, L}(S, t)+M_{T_{0}} \\
& +\int_{t}^{T_{0}} e^{-r(v-t)}\left(C_{t}^{A, L}+\mathbb{L}_{S} C^{A, L}-r C^{A, L}\right)\left(S_{v}, v\right) d v \\
& +\frac{1}{2} \int_{t}^{T_{0}} e^{-r(v-t)} \Delta_{S} C^{A, L}\left(B^{L, 1}(v), v\right) 1_{\left\{S_{v}=B^{L, 1}(v)>L_{1}\right\}} d \ell_{v}^{B^{L, 1}} \\
& +\frac{1}{2} \int_{t}^{T_{0}} e^{-r(v-t)} \Delta_{S} C^{A, L}\left(B^{L, 1}(v), v\right) 1_{\left\{S_{v}=B^{L, 1}(v)=L_{1}\right\}} d \ell_{v}^{B^{L, 1}} \\
& +\frac{1}{2} \int_{t}^{T_{0}} e^{-r(v-t)} \Delta_{S} C^{A, L}\left(L_{1}, v\right) 1_{\left\{S_{v}=L_{1}<B^{L, 1}(v)\right\}} d \ell_{v}^{L_{1}} \\
& =C^{A, L}(S, t)+M_{T_{0}}+\int_{t}^{T_{0}} e^{-r(v-t)} h_{2} 1_{\left\{S_{v} \in\left(L_{1}, B^{L, 1}(v)\right)\right\}} d v \\
& +\frac{1}{2} \int_{t}^{T_{0}} e^{-r(v-t)} C_{S}^{A, L}\left(B^{L, 1}(v)+, v\right) 1_{\left\{S_{v}=B^{L, 1}(v)\right\}} d \ell_{v}^{B^{L, 1}} \\
& -\frac{1}{2} \int_{t}^{T_{0}} e^{-r(v-t)} C_{S}^{A, L}\left(L_{1}-, v\right) 1_{\left\{S_{v}=L_{1}\right\}} d \ell_{v}^{L_{1}} \\
& =C^{A, L}(S, t)+M_{T_{0}}+\int_{t}^{T_{0}} e^{-r(v-t)} h_{2} 1_{\left\{S_{v} \in\left(L_{1}, B^{L, 1}(v)\right)\right\}} d v \\
& +\frac{1}{2} \int_{t}^{T_{0}} e^{-r(v-t)} C_{S}^{A, L}\left(B^{L, 1}(v)+, v\right) d \ell_{v}^{B^{L, 1}}
\end{aligned}
$$




$$
-\frac{1}{2} \int_{t}^{T_{0}} e^{-r(v-t)} C_{S}^{A, L}\left(L_{1}-, v\right) d \ell_{v}^{L_{1}}
$$

where $\Delta_{S} C^{A, L}(S, t) \equiv C_{S}^{A, L}(S+, t)-C_{S}^{A, L}(S-, t)$ is the jump of the derivative of $C^{A, L}$ at $S>0$ for $t \in\left[0, T_{0}\right) ; \ell^{B^{L, 1}}$ and $\ell^{L_{1}}$ are the local times that $S$ spends at $B^{L, 1}$ and $L_{1}$, respectively; $M=\left(M_{s}\right)_{s \geq t}$ is the martingale term, and we exploited (4.12) with (4.14). We also used that $C_{S}^{A, L}\left(B^{L, 1}(t)-, t\right)=0$ and $C_{S}^{A, L}\left(L_{1}+, t\right)=0$ for $t \in\left[0, T_{0}\right)$ when $B^{L, 1}(t)>L_{1}$. Note that $C^{A, L}(S, t)$ is known for $S<L_{1}$ and $t \in\left[0, T_{0}\right)$ as the optimal exercise rule is to wait until we hit $L_{1}$ before $T_{0}$, otherwise we obtain the value $C^{0}\left(S_{T_{0}}, T_{0}\right)$, i.e.,

$$
C^{A, L}(S, t)=\left(L_{1}-K\right) \mathrm{E}_{t}\left[e^{-r\left(\tau_{L_{1}}-t\right)} 1_{\left\{\tau_{L_{1}}<T_{0}\right\}}\right]+\mathrm{E}_{t}\left[e^{-r\left(T_{0}-t\right)} C^{0}\left(S_{T^{0}}, T_{0}\right) 1_{\left\{\tau_{L_{1}} \geq T_{0}\right\}}\right]
$$

where $\tau_{L_{1}}=\inf \left\{u \geq t: S_{u}=L_{1}\right\}$ denotes the first hitting time of $L_{1}$ when $S_{t}=S<L_{1}$ (see Lemma 8.1 in Appendix for the formulas for both expectations).

Now, taking expectation $\mathrm{E}_{t}$, using the optional sampling theorem, the terminal condition $C^{A, L}\left(S, T_{0}\right)=C^{0}\left(S, T_{0}\right)$ for $S>0$, the formula (2.14) in terms of local times at $L_{1}$ and $B^{L, 1}$, and rearranging terms in (4.20), we obtain the early exercise premium representation below.

Theorem 4.7. The price of the American capped call option has the EEP representation

$$
C^{A, L}(S, t)=C^{E, L}(S, t)+\Pi\left(S, t ; B^{L, 1}(\cdot)\right)
$$

for $S>0$ and $t<T_{0}$ where

$$
\begin{aligned}
& C^{E, L}(S, t)=\mathrm{E}_{t}\left[e^{-r\left(T_{0}-t\right)} C^{0}\left(S_{T_{0}}, T_{0}\right)\right] \\
& \Pi\left(S, t ; B^{L, 1}(\cdot)\right)= \\
& =r\left(L_{1}-K\right) \int_{t}^{T_{0}} e^{-r(v-t)} \mathrm{E}_{t}\left[1_{\left\{S_{v} \in\left(L_{1}, B^{L, 1}(v)\right)\right\}}\right] d v \\
& \quad-\frac{1}{2} \int_{t}^{T_{0}} e^{-r(v-t)} C_{S}^{A, L}\left(B^{L, 1}(v)+, v\right) \varphi\left(-\frac{\log \left(B^{L, 1}(v) / S\right)-\left(r-\delta-\frac{\sigma^{2}}{2}\right)(v-t)}{\sigma \sqrt{v-t}}\right) \frac{\sigma B^{L, 1}(v)}{\sqrt{v-t}} d v \\
& \quad+\frac{1}{2} \int_{t}^{T_{0}} e^{-r(v-t)} C_{S}^{A, L}\left(L_{1}-, v\right) \varphi\left(-\frac{\log \left(L_{1} / S\right)-\left(r-\delta-\frac{\sigma^{2}}{2}\right)(v-t)}{\sigma \sqrt{v-t}}\right) \frac{\sigma L_{1}}{\sqrt{v-t}} d v .
\end{aligned}
$$

In this expression, $C^{E, L}(S, t)$ is the price of the European derivative with payoff $C^{0}\left(S_{T_{0}}, T_{0}\right)$ at the maturity date $T_{0}$ and $\Pi\left(S, t ; B^{L, 1}(\cdot)\right)$ is the early exercise premium given the optimal exercise boundary $B^{L, 1}$.

8. To characterize the optimal exercise boundary $B^{L, 1}=\left(B^{L, 1}(t)\right)_{t \in\left[0, T_{0}\right]}$, we insert $S=$ $B^{L, 1}(t)$ for $t \in\left(t^{0} \vee 0, T_{0}\right)$ into (4.22) and use (4.15) to derive the following recursive integral equation for $B^{L, 1}$

$$
L_{1}-K=C^{E, L}\left(B^{L, 1}(t), t\right)+\Pi\left(B^{L, 1}(t), t ; B^{L, 1}(\cdot)\right)
$$

for $t \in\left(t^{0} \vee 0, T_{0}\right)$, subject to the boundary condition $B^{L, 1}\left(T_{0}-\right)=L_{1}$. This completes the proof of Theorem $3.1(i)$. 


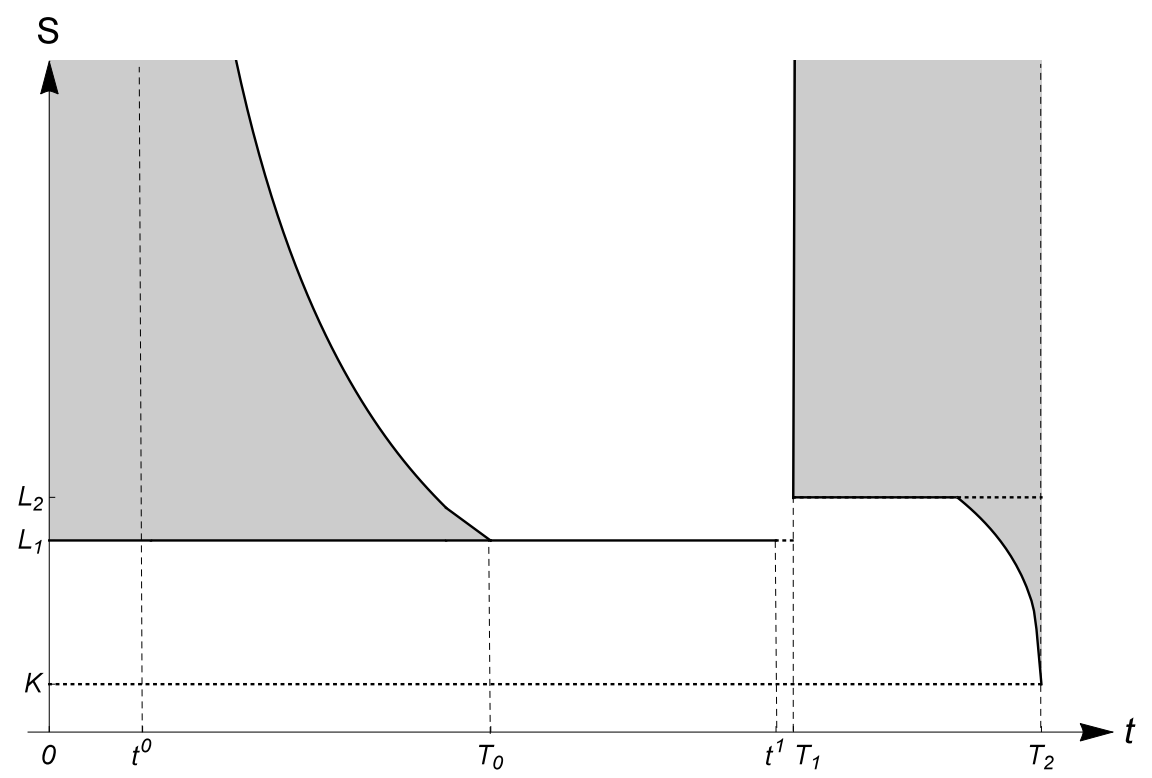

Figure 6. This figure plots the immediate exercise region (gray) $\mathcal{E}$ in problem (2.3). The part $\mathcal{E}_{1}$ of the exercise region on $\left[0, T_{1}\right.$ ) consists of $\widetilde{\mathcal{E}}_{1}$ (with upper boundary $B^{L, 1}$ on $\left.\left[0, T_{0}\right]\right)$ and the horizontal segment $\overline{\mathcal{E}}_{1}=\left\{(S, t) \in \mathbb{R}^{+} \times\left[T_{0}, t^{1}\right]\right.$ : $\left.S=L_{1}\right\}$. The parameter set is $T_{1}=3, T_{2}=4, K=1, L_{1}=1.3, L_{2}=1.39, r=$ $0.1, \delta=0.1, \sigma=0.3$. We obtain that $t^{0}=0.22, T_{0}=1.78$ and $t^{1}=2.93$.

Remark 4.8. It is important to note that the integral equation (4.25) for $B^{L, 1}$ is implicit, as it depends on the derivatives $C_{S}^{A, L}\left(L_{1}-, v\right)$ and $C_{S}^{A, L}\left(B^{L, 1}(v)+, v\right)$ of $C^{A, L}$ at $L_{1}$ and $B^{L, 1}$. As mentioned above, the former derivative can be estimated independently of $B^{L, 1}$ using (4.21). However, the latter one requires unknown values of $C^{A, L}$ above $L_{1}$. To tackle this problem numerically, we use backward induction with a quadrature scheme to approximate $\Pi$. Then, at each time step $v$, we obtain the value of the boundary $B^{L, 1}(v)$ as the solution to an algebraic equation and also $C_{S}^{A, L}\left(B^{L, 1}(v)+, v\right)$ using (4.22) as we already recovered $B^{L, 1}(u)$ for $u>v$ (see details in Section 7). We also note that we could avoid the presence of $C_{S}^{A, L}\left(B^{L, 1}(v)+, v\right)$ by imposing the smooth-fit condition at $B^{L, 1}$ (see Remark 4.6) and numerical results seem to provide support for this condition. However, as we would like to keep the analysis as general and rigorous as possible, we do not impose it.

\section{Case $B\left(T_{1}\right) \leq L_{1}<L_{2}$}

Let us now assume that $L_{1} \geq B\left(T_{1}\right)$, i.e., $t^{*}<T_{1}$. Note that under this assumption we have that $B^{L, 2}(t)=B(t)$ for $t \in\left[T_{1}, T_{2}\right]$. It appears that this case is easier to tackle than the one in the previous section. The proof of this case is also divided into several steps.

Proof of Theorem 3.1 $(\mathrm{ii})$. 1. We start from the following observations on the structure of the exercise region on $\left[0, T_{1}\right]$. If $B(t) \leq S<L_{1}$ for $t \in\left[t^{*}, T_{1}\right)$, then it is optimal to immediately exercise the capped option at $(S, t)$. Indeed, we recall that $C^{A, L} \leq C^{A}$ and $C^{A}(S, t)=S-K$ 


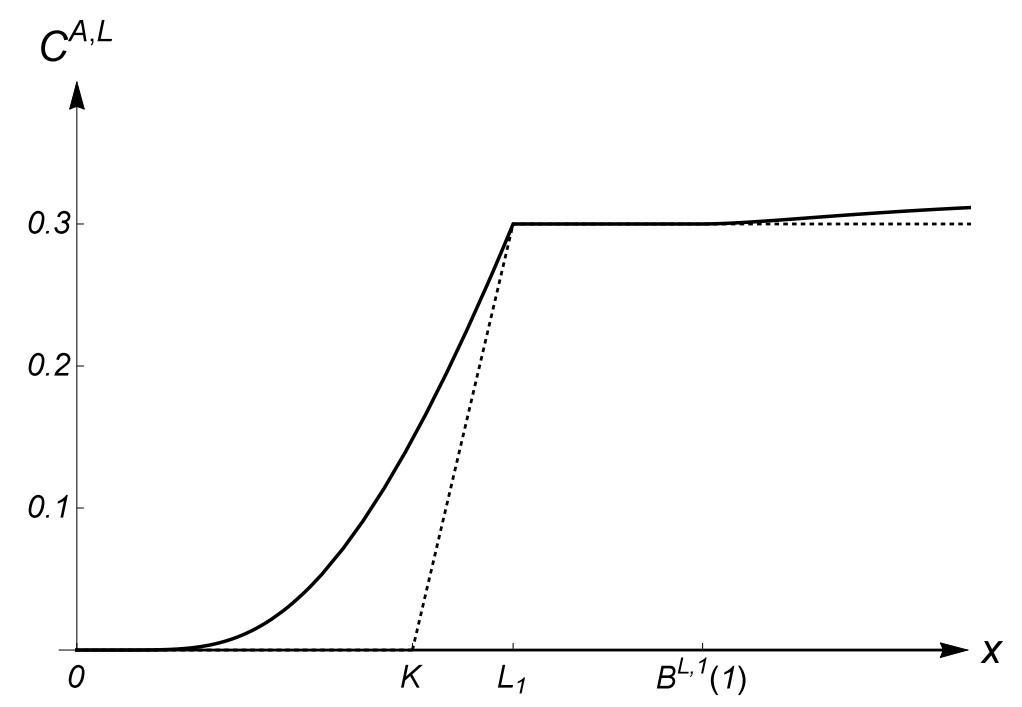

Figure 7. This figure plots the American capped option price $C^{A,}(S, 1)$ in (2.3). The dotted line is the immediate payoff $\left(S \wedge L_{1}-K\right)^{+}$. The parameter set is $T_{1}=3, T_{2}=4, K=1, L_{1}=1.3, L_{2}=1.39, r=0.1, \delta=0.1, \sigma=0.3$.

for $S \geq B(t)$. Then we have that $S \wedge L_{1}-K=S-K \leq C^{A, L}(S, t) \leq C^{A}(S, t)=S-K$. Therefore, $C^{A, L}(S, t)=S \wedge L_{1}-K$ which means that $(S, t)$ belongs to the exercise region.

Next, it is clear that $(S, t)$ belongs to the continuation region for $S<\min \left(L_{1}, B(t)\right)$ and $t \in\left[0, T_{1}\right)$. To prove it, the standard arguments with shorter maturity option can be applied (see, e.g., the proof of Lemma 4.1 or Broadie and Detemple (1995)). Using exactly the same arguments as in the previous section (see the proof of Theorem $4.2(\mathrm{ii})$ ), we can also prove that the exercise subregion is down-connected above $L_{1}$.

Now we show that it is optimal to exercise at $\left(L_{1}, t\right)$ for all $t \in\left[0, T_{1}\right]$. Indeed, it is already known that $\left(L_{1}, t\right) \in \mathcal{E}$ for all $t \in\left[t^{*}, T_{1}\right]$. Now let us fix any $t<t^{*}$ and use the same idea as in the proof of Theorem 4.2 (iii). We choose the auxiliary capped option with new maturity $\widetilde{T}_{1}=t^{*} \leq T_{1}$ for the cap $L_{1}$ so that $B\left(\widetilde{T}_{1}\right)=L_{1}$. Obviously, the price of the option increases. Also, we have that $\widetilde{t}^{1}$ (defined as in the previous section) equals $\widetilde{T}_{1}$. Thus, using the result of Theorem $4.2(\mathrm{iii})$, we know that $\left(L_{1}, t\right)$ belongs to the exercise region of the auxiliary option. As the price of this option is greater than that of the original and their payoffs coincide at $\left(L_{1}, t\right)$, we can conclude that $\left(L_{1}, t\right) \in \mathcal{E}_{1}$.

We then notice that $C^{A, L}(S, t)$ coincides with the price of the capped option $C^{A, L_{1}}(S, t)$ with single cap $L_{1}$ for $S \leq L_{1}$ and $t<T_{2}$. This due to the fact that both contracts have the same optimal exercise policy and the same payoff below $L_{1}$.

The next observation is that there exists $T_{0}<T_{1}$ such that $(S, t)$ belongs to the continuation region for all $S>L_{1}$ and $t \in\left[T_{0}, T_{1}\right)$. To show this, for $S>L_{1}$ and $t \in\left[0, T_{1}\right)$ let us consider the policy of waiting until $T_{1}$ unless the stock price hits the cap $L_{1}$. The value of this strategy is then given by

$$
C^{0}(S, t) \equiv\left(L_{1}-K\right) \mathrm{E}_{t}\left[e^{-r\left(\tau_{L_{1}}-t\right)} 1_{\left\{\tau_{L_{1}}<T_{1}\right\}}\right]+\mathrm{E}_{t}\left[e^{-r\left(T_{1}-t\right)}\left(S_{T_{1}} \wedge L_{2}-K\right) 1_{\left\{\tau_{L_{1}} \geq T_{1}\right\}}\right]
$$




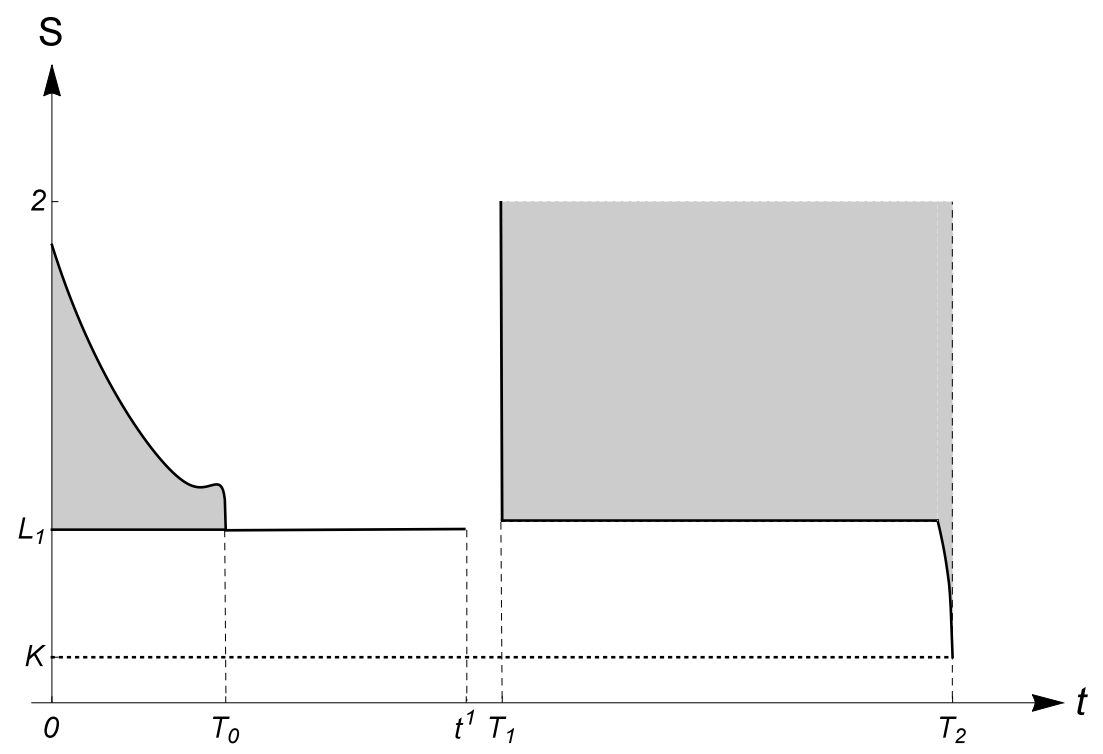

Figure 8. This figure plots the immediate exercise region (gray) $\mathcal{E}$ in problem (2.3). The part $\mathcal{E}_{1}$ of the exercise region on $\left[0, T_{1}\right.$ ) consists of $\widetilde{\mathcal{E}}_{1}$ (with upper boundary $B^{L, 1}$ on $\left.\left[0, T_{0}\right]\right)$ and the horizontal segment $\overline{\mathcal{E}}_{1}=\left\{(S, t) \in \mathbb{R}^{+} \times\left[T_{0}, t^{1}\right]\right.$ : $\left.S=L_{1}\right\}$. The exercise region on $\left[T_{1}, T_{2}\right)$ is characterized by $B^{L, 2}$, see Theorem $3.2(i)$. The parameter set is $T_{1}=1, T_{2}=2, K=1, L_{1}=1.28, L_{2}=1.3, r=$ $0.05, \delta=0.05, \sigma=0.5$. We obtain that $T_{0}=0.386$ and $t^{1}=0.988$. It can be seen that the upper boundary $B^{L, 1}$ is not decreasing everywhere.

where $\tau_{L_{1}}$ is the first hitting time of the cap $L_{1}$. In fact, this is the price of the European capped barrier down-and-out call option with rebate payoff $L_{1}-K$ at the barrier $L_{1}$. Direct examination of its well-known expression shows that the slope $C_{S}^{0}\left(L_{1}+, t\right)$ at $L_{1}$ converges to 1 as $t \rightarrow T_{1}$. Due to its continuity, for $t$ sufficiently close to $T_{1}$, the derivative at $L_{1}$ is still positive. We then define $T_{0}$ as the largest positive solution to the equation $C_{S}^{0}\left(L_{1}, t\right)=0$ if one exists; otherwise, $T_{0}=0$. As the slope is positive for $t \in\left(T_{0}, T_{1}\right)$, we have that $C^{0}(S, t)>L_{1}-K$ and it is optimal to wait at $(S, t)$ for $S>L_{1}$.

The option price at $T_{0}$ then reads

$$
C^{A, L}\left(S, T_{0}\right)=C^{A, L_{1}}\left(S, T_{0}\right)
$$

for $S \leq L_{1}$ and

$$
C^{A, L}\left(S, T_{0}\right)=C^{0}\left(S, T_{0}\right)
$$

for $S>L_{1}$ and where $\tau_{L_{1}}$ is the first hitting time of the cap $L_{1}$.

Finally, if $t^{0} \equiv T_{1}-\frac{1}{r} \log \left(\left(L_{2}-K\right) /\left(L_{1}-K\right)\right) \geq 0$, then $(S, t) \in \mathcal{E}_{1}$ for any $t \in\left[0, t^{0}\right]$ and $S \geq L_{1}$. The proof is the same as in the previous case.

2. The results above motivate us to define the upper exercise boundary $B^{L, 1}=\left(B^{L, 1}(t)\right)_{t \in\left[0, T_{1}\right)}$ such that the exercise subregion $\mathcal{E}_{1}$ on $\left[0, T_{1}\right)$ is given as

$$
\mathcal{E}_{1}=\left\{(S, t) \in \mathbb{R}^{+} \times\left[0, T^{1}\right): L_{1} \leq S \leq B^{L, 1}(t)\right\}
$$




$$
\cup\left\{(S, t) \in \mathbb{R}^{+} \times\left[t^{*}, T^{1}\right): B(t) \leq S \leq L_{1}\right\} .
$$

It is clear that $B^{L, 1}=L_{1}$ for $t \in\left[T_{0}, T_{1}\right)$ and $B^{L, 1}(t)=+\infty$ for $t \in\left[0, t^{0}\right]$ if $t^{0} \geq 0$.

As we know the optimal exercise policy on $\left[T_{0}, T_{2}\right]$, we set the new maturity date as $T_{0}$. Hence, the American capped option (2.3) is equivalent to an American capped derivative with maturity date $T_{0}$ and exercise payoff

$$
G\left(S_{\tau}, \tau\right)=\left(S_{\tau} \wedge L_{1}-K\right)^{+} 1_{\left\{\tau<T_{0}\right\}}+C^{A, L}\left(S_{T_{0}}, T_{0}\right) 1_{\left\{\tau=T_{0}\right\}}
$$

at $\tau \in\left[0, T_{0}\right]$ where $C^{A, L}\left(S, T_{0}\right)$ is given in (5.1)-(5.3). The equivalent contract has a constant cap $L_{1}$ on the interval $\left[0, T_{0}\right)$ and a known terminal value at $T_{0}$.

For this contract, the instantaneous benefits of waiting to exercise are given by

$$
H(S) \equiv\left(\mathbb{L}_{S} G-r G\right)(S)
$$

for $t<T_{0}$ and for $S>0$, and thus

$$
H(S)=h_{1}(S) 1_{\left\{K \leq S<L_{1}\right\}}+h_{2} 1_{\left\{S \geq L_{1}\right\}}
$$

where

$$
h_{1}(S)=(r-\delta) S-r(S-K)=r K-\delta S \quad \text { and } \quad h_{2}=-r\left(L_{1}-K\right) .
$$

Using standard arguments we obtain that the option price $C^{A, L}=C^{A, L}(S, t)$ solves PDE

$$
C_{t}^{A, L}+\mathbb{L}_{S} C^{A, L}-r C^{A, L}=0
$$

in the continuation set $\mathcal{C}_{1}=\left\{(S, t) \in \mathbb{R}^{+} \times\left[0, T_{0}\right]: S<\min \left(L_{1}, B(t)\right)\right.$ or $\left.S>B^{L, 1}(t)\right\}$.

3. We apply the local time-space formula on curves (again assuming that $B^{L, 1}$ is continuous and of bounded variation on $\left.\left[0, T_{0}\right]\right)$ to obtain

$$
\begin{aligned}
e^{-r\left(T_{0}-t\right)} & C^{A, L}\left(S_{T_{0}}, T_{0}\right) \\
= & C^{A, L}(S, t)+M_{T_{0}} \\
& +\int_{t}^{T_{0}} e^{-r(v-t)}\left(C_{t}^{A, L}+\mathbb{L}_{S} C^{A, L}-r C^{A, L}\right)\left(S_{v}, v\right) d v \\
& +\frac{1}{2} \int_{t}^{T_{0}} e^{-r(v-t)} \Delta_{S} C^{A, L}\left(B^{L, 1}(v), v\right) 1_{\left\{S_{v}=B^{L, 1}(v)>L_{1}\right\}} d \ell_{v}^{B^{L, 1}} \\
& +\frac{1}{2} \int_{t}^{T_{0}} e^{-r(v-t)} \Delta_{S} C^{A, L}\left(B^{L, 1}(v), v\right) 1_{\left\{S_{v}=B^{L, 1}(v)=L_{1}\right\}} d \ell_{v}^{B^{L, 1}} \\
& +\frac{1}{2} \int_{t}^{T_{0}} e^{-r(v-t)} \Delta_{S} C^{A, L}\left(L_{1}, v\right) 1_{\left\{S_{v}=L_{1}<B^{L, 1}(v)\right\}} d \ell_{v}^{L_{1}} \\
= & C^{A, L}(S, t)+M_{T_{0}}+\int_{t}^{T_{0}} e^{-r(v-t)} h_{1}\left(S_{v}\right) 1_{\left\{S_{v} \in\left(B(v), L_{1}\right)\right\}} d v \\
& +\int_{t}^{T_{0}} e^{-r(v-t)} h_{2} 1_{\left\{S_{v} \in\left(L_{1}, B^{L, 1}(v)\right)\right\}} d v
\end{aligned}
$$




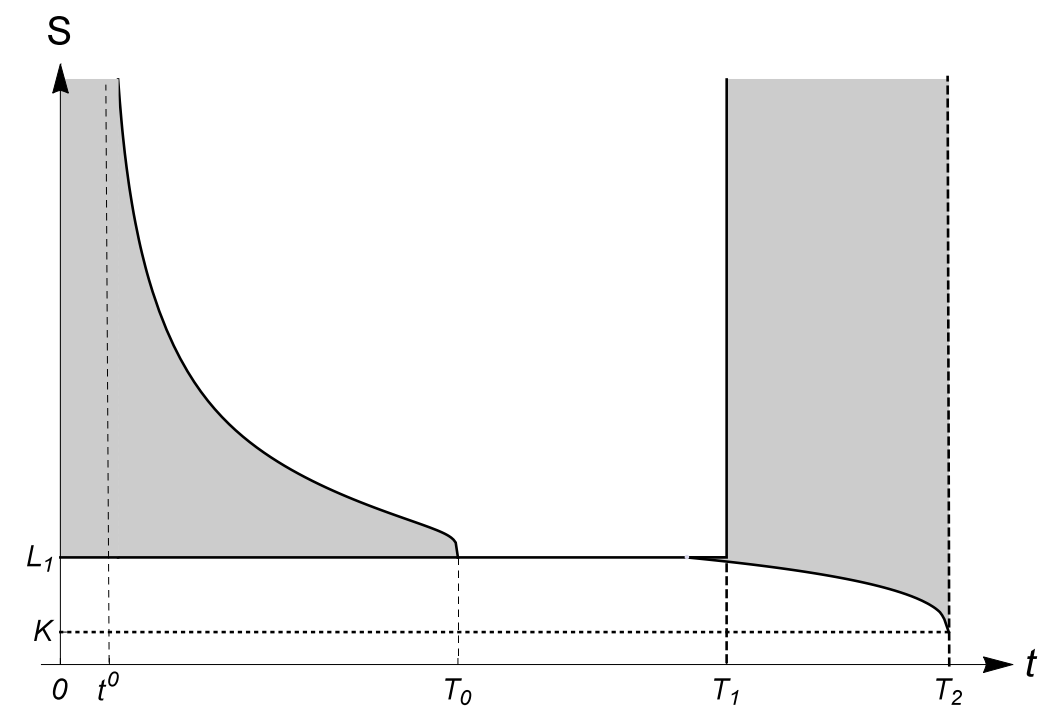

Figure 9. This figure plots the immediate exercise region (gray) $\mathcal{E}$ in problem (2.3) when $B\left(T_{1}\right) \leq L_{1}<L_{2}$. The parameter set is $T_{1}=3, T_{2}=4, K=1, L_{1}=$ $1.46, L_{2}=1.5, r=0.03, \delta=0.05, \sigma=0.25$. We obtain that $t^{0}=0.22$ and $T_{0}=1.79$.

$$
\begin{aligned}
& +\frac{1}{2} \int_{t}^{T_{0}} e^{-r(v-t)} C_{S}^{A, L}\left(B^{L, 1}(v)+, v\right) 1_{\left\{S_{v}=B^{L, 1}(v)\right\}} d \ell_{v}^{B^{L, 1}} \\
& -\frac{1}{2} \int_{t}^{T_{0}} e^{-r(v-t)} C_{S}^{A, L}\left(L_{1}-, v\right) 1_{\left\{S_{v}=L_{1}\right\}} d \ell_{v}^{L_{1}} \\
= & C^{A, L}(S, t)+M_{T_{0}}+\int_{t}^{T_{0}} e^{-r(v-t)} h_{1}\left(S_{v}\right) 1_{\left\{S_{v} \in\left(B(v), L_{1}\right)\right\}} d v \\
& +\int_{t}^{T_{0}} e^{-r(v-t)} h_{2} 1_{\left\{S_{v} \in\left(L_{1}, B^{L, 1}(v)\right)\right\}} d v \\
& +\frac{1}{2} \int_{t}^{T_{0}} e^{-r(v-t)} C_{S}^{A, L}\left(B^{L, 1}(v)+, v\right) d \ell_{v}^{B^{L, 1}} \\
& -\frac{1}{2} \int_{t}^{T_{0}} e^{-r(v-t)} C_{S}^{A, L}\left(L_{1}-, v\right) d \ell_{v}^{L_{1}}
\end{aligned}
$$

where $M=\left(M_{s}\right)_{s>t}$ is the martingale term and we used (5.7) and (5.9). We also used that $C_{S}^{A, L}\left(B^{L, 1}(t)-, t\right)=0$ and $C_{S}^{A, L}\left(L_{1}+, t\right)=0$ for $t \in\left[0, T_{0}\right)$ when $B^{L, 1}(t)>L_{1}$. We recall that $C^{A, L}(S, t)=C^{A, L_{1}}(S, t)$ for $S<L_{1}$ and $t \in\left[0, T_{1}\right)$.

Now, taking expectation $\mathrm{E}_{t}$, using the optional sampling theorem, the terminal condition (5.2)-(5.3), rearranging terms in (5.10), we obtain the early exercise premium representation below.

Theorem 5.1. The price of the American capped call option has the EEP representation

$$
C^{A, L}(S, t)=C^{E, L}(S, t)+\Pi\left(S, t ; B^{L, 1}(\cdot)\right)
$$


for $S>0$ and $t<T_{0}$ where

$$
\begin{aligned}
& C^{E, L}(S, t)=\mathrm{E}_{t}\left[e^{-r\left(T_{0}-t\right)} C^{A, L}\left(S_{T_{0}}, T_{0}\right)\right] \\
& \Pi\left(S, t ; B^{L, 1}(\cdot)\right)= \\
& =\int_{t}^{T_{0}} e^{-r(v-t)} \mathrm{E}_{t}\left[\left(\delta S_{v}-r K\right) 1_{\left\{S_{v} \in\left(B(v), L_{1}\right)\right\}}\right] d v \\
& \quad+r\left(L_{1}-K\right) \int_{t}^{T_{0}} e^{-r(v-t)} \mathrm{E}_{t}\left[1_{\left.\left\{S_{v} \in\left(L_{1}, B^{L, 1}(v)\right)\right\}\right] d v}\right. \\
& \quad-\frac{1}{2} \int_{t}^{T_{0}} e^{-r(v-t)} C_{S}^{A, L}\left(B^{L, 1}(v)+, v\right) \varphi\left(-\frac{\log \left(B^{L, 1}(v) / S\right)-\left(r-\delta-\frac{\sigma^{2}}{2}\right)(v-t)}{\sigma \sqrt{v-t}}\right) \frac{\sigma B^{L, 1}(v)}{\sqrt{v-t}} d v \\
& \quad+\frac{1}{2} \int_{t}^{T_{0}} e^{-r(v-t)} C_{S}^{A, L_{1}}\left(L_{1}-, v\right) \varphi\left(-\frac{\log \left(L_{1} / S\right)-\left(r-\delta-\frac{\sigma^{2}}{2}\right)(v-t)}{\sigma \sqrt{v-t}}\right) \frac{\sigma L_{1}}{\sqrt{v-t}} d v .
\end{aligned}
$$

In this expression, $C^{E, L}(S, t)$ is the price of the European derivative with payoff $C^{A, L_{2}}\left(S_{T_{0}}, T_{0}\right)$ at the maturity date $T_{0}$ and $\Pi\left(S, t ; B^{L, 1}(\cdot)\right)$ is the early exercise premium given the optimal exercise boundary $B^{L, 1}$.

4. To characterize the optimal exercise boundary $B^{L, 1}=\left(B^{L, 1}(t)\right)_{t \in\left[0, T_{0}\right]}$, we insert $S=$ $B^{L, 1}(t)$ for $t \in\left(t^{0} \vee 0, T_{0}\right)$ into (5.11) to derive the following recursive integral equation for $B^{L, 1}$

$$
L_{1}-K=C^{E, L}\left(B^{L, 1}(t), t\right)+\Pi\left(B^{L, 1}(t), t ; B^{L, 1}(\cdot)\right)
$$

for $t \in\left(t^{0} \vee 0, T_{0}\right)$, subject to the boundary condition $B^{L, 1}\left(T_{0}-\right)=L_{1}$. This completes the proof of Theorem $3.1(i i)$.

\section{Case $L_{1}>L_{2}$}

Finally, we consider the case $L_{1}>L_{2}$ and assume that the cap is left-continuous

$$
L_{\tau}=L_{1} 1_{\tau \leq T_{1}}+L_{2} 1_{T_{1}<\tau \leq T_{2}} .
$$

In this case we already know the optimal exercise rule on the interval $\left(T_{1}, T_{2}\right]$.

Proof of Theorem 3.1 $($ iii $)$. 1. We first note that the value of the option at $T_{1}$ is given as

$$
C^{A, L}\left(S, T_{1}\right)=G\left(S, T_{1}\right) \equiv \max \left(S \wedge L_{1}-K, C^{A, L_{2}}\left(S, T_{1}\right)\right)
$$

for $S>0$ as we decide whether to exercise it immediately and receive $S \wedge L_{1}-K$ or continue and get $C^{A, L_{2}}\left(S, T_{1}\right)$. Using that $L_{1}>L_{2}$ and the known structure of $C^{A, L_{2}}$ we may rewrite the option price as

$$
C^{A, L}\left(S, T_{1}\right)=\left(S \wedge L_{1}-K\right) 1_{\left\{S \geq L_{2} \wedge B\left(T_{1}\right)\right\}}+C^{A, L_{2}}\left(S, T_{1}\right) 1_{\left\{S<L_{2} \wedge B\left(T_{1}\right)\right\}}
$$

for $S>0$. We also note that the payoff $G(S, t) \equiv\left(S \wedge L_{1}-K\right)^{+}$at $t \in\left[0, T_{1}\right)$ is dominated by the value at $T_{1}$ and thus we can apply the results of Palczewski and Stettner (2010) to 
conclude that $C^{A, L}$ is continuous on $\mathbb{R}^{+} \times\left[0, T_{1}\right]$ and that the optimal exercise time on $\left[0, T_{1}\right]$ is given in the standard form

$$
\tau_{*}=\inf \left\{t>0: C^{A, L}\left(S_{t}, t\right)=G\left(S_{t}, t\right)\right\} .
$$

One can also see that the value function $C^{A, L}(S, t)$ is discontinuous from the right at $T_{1}+$ for $S>L_{2} \wedge B\left(T_{1}\right)$. This is not crucial, as we concentrate on the interval $\left[0, T_{1}\right]$.

2. We now note that, in this case, the cap $L$ is non-increasing on $\left[0, T_{2}\right]$ and thus the option price $C^{A, L}(S, t)$ is decreasing in $t$ for fixed $S>0$. Therefore, the exercise region is right-connected. Standard dominance arguments show that the exercise region is up-connected below the cap level $L_{1}$ on $\left[0, T_{1}\right)$. The next observation is that it is optimal to exercise at $(S, t)$ for $S \geq L_{1}$ and $t \in\left[0, T_{1}\right]$, as one attains the maximum possible payoff there. It is also clear that the local benefits of waiting to exercise are positive when $S<r K / \delta$, so the holder should not exercise the option below $r K / \delta$ prior to $T_{1}$.

Consequently, we can define the non-increasing exercise boundary $B^{L, 1}=\left(B^{L, 1}(t)\right)_{t \in\left[0, T_{1}\right]}$ such that the exercise subregion $\mathcal{E}_{1}$ on $\left[0, T_{1}\right]$ is given as

$$
\mathcal{E}_{1}=\left\{(S, t) \in \mathbb{R}^{+} \times\left[0, T^{1}\right]: S \geq B^{L, 1}(t)\right\} .
$$

From the arguments above and (6.3) , we have that $B^{L, 1}\left(T_{1}-\right)=\max \left(r K / \delta, L_{2} \wedge B\left(T_{1}\right)\right)$ and $B^{L, 1}\left(T_{1}\right)=L_{2} \wedge B\left(T_{1}\right)$. Therefore the boundary $B^{L, 1}$ may exhibit a jump at $T_{1}$.

We define time $t_{1}^{*}$ as the root of the equation $B^{L, 1}(t)=L_{1}$ on $\left[0, T_{1}\right)$. If $B^{L, 1}<L_{1}$ on $\left[0, T_{1}\right)$, then we let $t_{1}^{*}=0$. As we know the optimal exercise policy on $\left(T_{1}, T_{2}\right]$ we set the new maturity date as $T_{1}$. Hence, the American capped option (2.3) is equivalent to an American capped derivative with maturity date $T_{1}$ and exercise payoff

$$
G\left(S_{\tau}, \tau\right)=\left(S_{\tau} \wedge L_{1}-K\right)^{+} 1_{\left\{\tau<T_{1}\right\}}+G\left(S_{T_{1}}, T_{1}\right) 1_{\left\{\tau=T_{1}\right\}}
$$

at $\tau \in\left[0, T_{1}\right]$ where $G\left(S, T_{1}\right)$ is given in (6.3). We define the instantaneous benefits $H(S)$ of waiting to exercise, the function $h_{1}(S)$ and the constant $h_{2}$ as in the previous section. Also, the option price $C^{A, L}$ solves the PDE

$$
C_{t}^{A, L}+\mathbb{L}_{S} C^{A, L}-r C^{A, L}=0
$$

in the continuation set $\mathcal{C}_{1}=\left\{(S, t) \in \mathbb{R}^{+} \times\left[0, T_{1}\right): S<B^{L, 1}(t)\right\}$.

We recall that the boundary $B^{L, 1}$ is non-increasing and this fact allows us to easily prove that the smooth-fit condition holds at $B^{L, 1}(t)$ for $t \in\left(t_{1}^{*}, T_{1}\right)$ (see e.g., p. 381, Section 25 in Peskir and Shiryaev (2006)). The monotonicity of $B^{L, 1}$ also enables us to show the continuity of $B^{L, 1}$ on $\left[0, T_{1}\right)$.

3. In this case, we can apply the local time-space formula on curves as the boundary $B^{L, 1}$ is continuous and of bounded variation so that we obtain

$$
\begin{aligned}
e^{-r\left(T_{1}-t\right)} & C^{A, L}\left(S_{T_{1}}, T_{1}\right) \\
= & C^{A, L}(S, t)+\int_{t}^{T_{1}} e^{-r(u-t)}\left(C_{t}^{A, L}+\mathrm{E}_{S} C^{A, L}-r C^{A, L}\right)\left(S_{u}, u\right) d u+M_{T_{1}} \\
& +\frac{1}{2} \int_{t}^{T_{1}} e^{-r(u-t)}\left(C_{S}^{A, L}\left(S_{u}+, u\right)-C_{S}^{A, L}\left(S_{u}-, u\right)\right) 1_{\left\{S_{u}=L_{1}\right\}} d \ell_{u}^{L_{1}}
\end{aligned}
$$




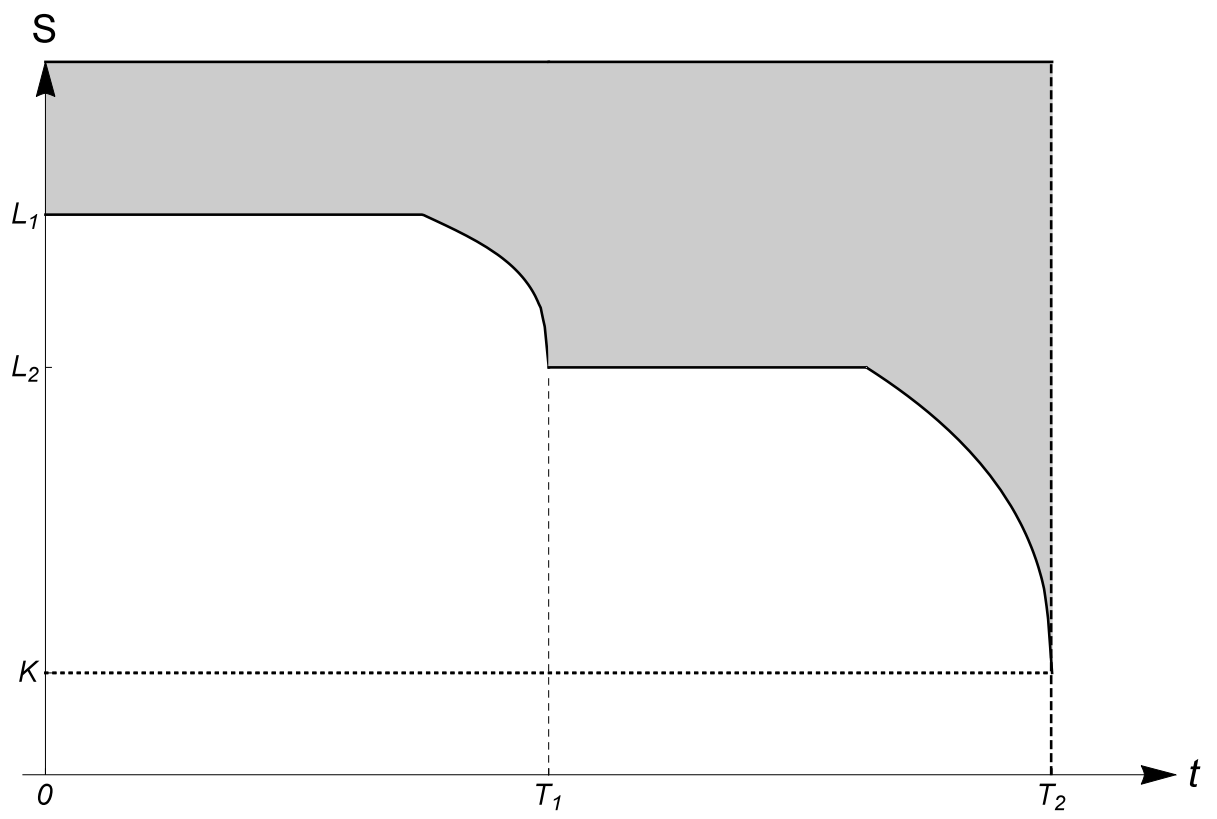

Figure 10. This figure plots the immediate exercise region (gray) $\mathcal{E}$ in problem (2.3) when $L_{1}>L_{2}$. The parameter set is $T_{1}=1, T_{2}=2, K=1, L_{1}=1.45, L_{2}=$ $1.3, r=0.03, \delta=0.05, \sigma=0.25$. We obtain that $t_{1}^{*}=0.75$ and $t^{*}=1.63$.

$$
\begin{aligned}
& +\frac{1}{2} \int_{t_{1}^{*} \vee t}^{T_{1}} e^{-r(u-t)}\left(C_{S}^{A, L}\left(S_{u}+, u\right)-C_{S}^{A, L}\left(S_{u}-, u\right)\right) 1_{\left\{S_{u}=B^{L, 1}(u)\right\}} d \ell_{u}^{B^{L, 1}} \\
= & C^{A, L}(S, t)+\int_{t_{1}^{*} \vee t}^{T_{1}} e^{-r(u-t)}\left(r K-\delta S_{u}\right) 1_{\left\{B^{L, 1}(u) \leq S_{u} \leq L_{1}\right\}} d u \\
& -r\left(L_{1}-K\right) \int_{t}^{T_{1}} e^{-r(u-t)} 1_{\left\{S_{u} \geq L_{1}\right\}} d u+M_{T_{1}} \\
& -\frac{1}{2} \int_{t}^{T_{1}} e^{-r(u-t)} C_{S}^{A, L}\left(L_{1}-, u\right) d \ell_{u}^{L_{1}}
\end{aligned}
$$

where $M=\left(M_{t}\right)_{t>0}$ is the martingale part and we used the PDE (6.7), the smooth-fit condition at $B^{L}$ on $\left(t_{1}^{*}, T_{1}\right)$, and that $C_{S}^{A, L}\left(L_{1}+, \cdot\right)=0$.

Now, taking expectation $\mathrm{E}_{t}$, using the optional sampling theorem, the terminal condition $C^{A, L}\left(S, T_{1}\right)=G\left(S, T_{1}\right)$ for $S>0$, rearranging terms in (6.8), we obtain the early exercise premium representation below.

Theorem 6.1. The price of the American capped call option has the EEP representation

$$
C^{A, L}(S, t)=C^{E, L}(S, t)+\Pi\left(S, t ; B^{L, 1}(\cdot)\right)
$$

for $S>0$ and $t<T_{1}$ where

$$
\begin{aligned}
& C^{E, L}(S, t)=\mathrm{E}_{t}\left[e^{-r\left(T_{1}-t\right)} G\left(S_{T_{1}}, T_{1}\right)\right] \\
& \Pi\left(S, t ; B^{L, 1}(\cdot)\right)=
\end{aligned}
$$




$$
\begin{aligned}
= & \int_{t \vee t_{1}^{*}}^{T_{1}} e^{-r(u-t)} \mathrm{E}_{t}\left[\left(\delta S_{u}-r K\right) 1_{\left\{S_{u} \in\left(B^{L, 1}(u), L_{1}\right)\right\}}\right] d u \\
& +r\left(L_{1}-K\right) \int_{t}^{T_{1}} e^{-r(u-t)} \mathrm{E}_{t}\left[1_{\left\{S_{u} \geq L_{1}\right\}}\right] d u \\
& +\frac{1}{2} \int_{t}^{T_{1}} e^{-r(u-t)} C_{S}^{A, L}\left(L_{1}-, u\right) \varphi\left(-\frac{\log \left(L_{1} / S\right)-\left(r-\delta-\frac{\sigma^{2}}{2}\right)(u-t)}{\sigma \sqrt{u-t}}\right) \frac{\sigma L_{1}}{\sqrt{u-t}} d u .
\end{aligned}
$$

In this expression, $C^{E, L}(S, t)$ is the price of the European derivative with payoff $G\left(S_{T_{1}}, T_{1}\right)$ at the maturity date $T_{1}$ and $\Pi\left(S, t ; B^{L, 1}(\cdot)\right)$ is the early exercise premium given the optimal exercise boundary $B^{L, 1}$. We note that $C_{S}^{A, L}\left(L_{1}-, u\right)=1$ on $\left[t_{1}^{*}, T_{1}\right)$.

4. To characterize the optimal exercise boundary $B^{L, 1}=\left(B^{L, 1}(t)\right)_{t \in\left[0, T_{1}\right]}$, we insert $S=$ $B^{L, 1}(t)$ into (6.9) to derive the following recursive integral equation for $B^{L, 1}$

$$
B^{L, 1}(t)-K=C^{E, L}\left(B^{L, 1}(t), t\right)+\Pi\left(B^{L, 1}(t), t ; B^{L, 1}(\cdot)\right)
$$

for $t \in\left[t_{1}^{*}, T_{1}\right)$, subject to the boundary condition $B^{L, 1}\left(T_{1}-\right)=\max \left(r K / \delta, L_{2} \wedge B\left(T_{1}\right)\right)$.

Let us now briefly discuss the case of right-continuous cap $L$ with $L_{1}>L_{2}$. In this instance, we cannot employ the results of Palczewski and Stettner (2010) as the payoff jumps down at $T_{1}$. In fact, in some of the possible cases there is no optimal stopping time. For example, if $\delta$ is sufficiently small, then $r K / \delta>L_{2}$ and it is optimal to wait for $S \in\left(L_{2}, r K / \delta \wedge L_{1}\right)$ and $t<T_{1}$ as the local benefits of waiting are positive. However, at $t=T_{1}$, the payoff suddenly decreases from $S-K$ to $L_{2}-K$. The latter is the maximum possible payoff on $\left[T_{1} \cdot T_{2}\right]$. Thus, there is no optimal exercise rule in this case.

Standard arguments show that the values of both option versions (right-continuous and leftcontinuous caps) coincide at $t<T_{1}$ and $t>T_{1}$ for given $S>0$. The differences between two contracts are that (i) the right-continuous version may not have an optimal exercise time; (ii) the values at $T_{1}$ differ; (iii) the value function of the right-continuous version has a discontinuity from the left and that of the left-continuous version a discontinuity from the right at $T_{1}$. We also note that even though the right-continuous version does not have an optimal exercise time in some cases, one can construct a sequence of stopping times such that the corresponding value functions converge to the option price when $t<T_{1}$.

\section{Numerical Algorithm}

In this section, we describe the numerical solution to the American capped option problem (2.3) for all three cases: (i) $L_{1}<\min \left(L_{2}, B\left(T_{1}\right)\right)$; (ii) $B\left(T_{1}\right) \leq L_{1}<L_{2}$; (iii) $L_{1}>L_{2}$.

(i) Here we discuss the numerical algorithm for the case $L_{1}<\min \left(L_{2}, B\left(T_{1}\right)\right.$ ) using (4.22) and (4.25), and provide a step-by-step algorithm.

Step 1. We solve the well-known recursive integral equation for the boundary $B$ of the uncapped call option (for details of the numerical implementation, see, e.g., Chapter 8 of Detemple (2006)). This gives the early exercise premium representation for the uncapped option price $C^{A}(S, t)$.

Step 2. Using the formula (2.9), Lemma 8.1 and the EEP formula for $C^{A}$, we compute $C^{A, L_{2}}\left(L_{2}-\varepsilon, t\right)$ for any $t \in\left[0, t^{*}\right)$ and fixed small $\varepsilon>0$ to estimate $C_{S}^{A, L_{2}}\left(L_{2}-, t\right)$ (see Figure 
3). We then exploit (2.11) to compute $C^{A, L_{2}}\left(S, T_{1}\right)$ for any $S>0$ (see Figure 2). It is more efficient to use (2.11) and compute one-dimensional integrals rather than the formula (2.9), as the latter requires the integration of the call option price $C^{A}$, which is also given in an integral form.

Step 3. Here we compute the function $C^{w}(S, t)$ for $S>0$ and $t<T_{1}$ by using (4.1).

Step 4. We are now ready to compute $B^{w}(t)$ for all $t \in\left[0, T_{1}\right]$ using the function $C^{w}$ from Step 3 and the definition (4.3), and also to determine numerically $t^{1}$ (see Figure 4).

Step 5. Here, we identify $T_{0}$. For this, we compute $C^{0}\left(L_{1}+\varepsilon, t\right)$ for very small $\varepsilon>0$ and $t<t^{1}$ using (4.7), Lemma 8.1 and the values of $C^{w}$ at $t^{1}$ (see Step 3) to estimate $C_{S}^{0}\left(L_{1}+, t\right)$ (see Figure 5). Starting from $t=t^{1}$, we go backward in time and observe that $C^{0}\left(L_{1}+\varepsilon, t\right)>L_{1}-K$ if and only if $t>T_{0}$ for some $T_{0}<t^{1}$. Thus, we obtain $T_{0}$.

Step 6. Before solving the recursive integral equation (4.25) for $B^{L, 1}$, we estimate $C_{S}^{A, L}\left(L_{1}-, t\right)$ for $t \in\left[0, T_{0}\right)$ as $\frac{1}{\varepsilon}\left(L_{1}-K-C^{A, L}\left(L_{1}-\varepsilon, t\right)\right)$ for fixed small $\varepsilon>0$, where we use (4.21), Lemma 8.1 and values of $C^{0}$ to compute $C^{A, L}\left(L_{1}-\varepsilon, t\right)$.

Step \%. Finally, we have all ingredients to solve the integral equation (4.25) numerically. We discretize the interval $\left[0, T_{0}\right]$ with step $h$ and approximate the integrals by a quadrature scheme. Then, using the terminal condition $B^{L, 1}\left(T_{0}\right)=L_{1}$ and backward induction, we recover the boundary $B^{L, 1}$ at points $T_{0}-h, T_{0}-2 h, \ldots, 0$ by solving the sequence of algebraic equations. We also have to obtain $C_{S}^{A, L}\left(B^{L, 1}\left(T_{0}-i h\right)+, T_{0}-i h\right)$ at each time step, as it is required to compute $B^{L, 1}$. The derivative is estimated from (4.22) as we already computed $B^{L, 1}\left(T_{0}-j h\right)$ and $C_{S}^{A, L}\left(B^{L, 1}\left(T_{0}-j h\right)+, T_{0}-j h\right)$ for $1 \leq j<i$. Hence, we also need to estimate $C_{S}^{A, L}\left(L_{1}+, T_{0}\right)$, which simply equals $C_{S}^{0}\left(L_{1}+, T_{0}\right)$. Having interpolated and computed $B^{L, 1}$ on $\left[0, T_{0}\right]$ (see Figure 6 ), we can recover the American capped option price $C^{A, L}$ using (4.22) (see Figure 7).

Numerical examples.

Example 1. To illustrate the algorithm, we choose a parameters set and implement all the steps above to obtain the immediate exercise region and the price of the American capped call option. The parameters values are: $T_{1}=3, T_{2}=4, K=1, L_{1}=1.3, L_{2}=1.39, r=0.1, \delta=$ $0.1, \sigma=0.3$. For this set, we have that $T_{0}=1.78, t^{1}=2.93$ and $t^{*}=3.66$. We refer to Figures 1-7 for the graphical illustrations. Numerical experiments seem to provide evidence that the smooth pasting condition at $B^{L, 1}$ holds, so that $C_{S}^{A, L}\left(B^{L, 1}(v)+, v\right)=0$, and for this parameters set, the boundary $B^{L, 1}$ is decreasing.

Example 2. An interesting issue in this problem is the monotonicity of the upper boundary $B^{L, 1}$. There are two opposite factors that affect it: non-decreasing payoff and decreasing time horizon when $t$ increases. This tradeoff prevents us from proving the monotonicity of $B^{L, 1}$. In fact, for the set of parameters in Figure 8, we obtain that the boundary is non-monotone with some local periods of increasing behavior. This is another striking feature of the optimal stopping problem (2.3).

(ii) Now we turn to the case $B\left(T_{1}\right) \leq L_{1}<L_{2}$. As we saw in Section 5 , the structure of the exercise region is simpler than in the case $L_{1}<\min \left(L_{2}, B\left(T_{1}\right)\right)$ as $t^{1}=T_{1}$ and hence the numerical algorithm is less involved. In fact, we can skip Steps 3-4 from the algorithm above and only perform Steps 1,2,5,6 and 7 to solve (5.14). We illustrate this case in Figure 9 where we plot the immediate exercise region $\mathcal{E}$. 
(iii) Finally, we discuss the case $L_{1}>L_{2}$. As the exercise region is up-connected, the numerical algorithm becomes quite simple. The approach is similar to the algorithm for the classical uncapped call option boundary in Step 1. We simply discretize the interval $\left[0, T_{1}\right]$, then starting from $B^{L, 1}\left(T_{1}-\right)=\max \left(r K / \delta, L_{2} \wedge B\left(T_{1}\right)\right)$ we use backward induction and solve the sequence of algebraic equations to recover the exercise boundary $B^{L, 1}$ on $\left[0, T_{1}\right]$. We plot the immediate exercise region $\mathcal{E}$ in Figure 10.

\section{Appendix}

For the numerical computation of certain expectations appearing in the pricing formulas, we use the following auxiliary lemma.

Lemma 8.1. Let us fix some $0 \leq t<T$ and constant level $L>0$, and consider the first hitting time $\tau_{L}=\inf \left\{u \geq t: S_{u}=L\right\}$ of the level $L$ when the stock price starts at $S_{t}=S$ at time $t$. Then the following expectations can be computed as

$$
\mathrm{E}_{t}\left[e^{-r\left(\tau_{L}-t\right)} 1_{\left\{\tau_{L}<T\right\}}\right]=\left\{\begin{array}{l}
\lambda^{2 \phi / \sigma^{2}} \Phi\left(d_{0}\right)+\lambda^{2 \alpha / \sigma^{2}} \Phi\left(d_{0}+2 f \sqrt{T-t} / \sigma\right) \quad \text { for } S<L \\
\lambda^{2 \phi / \sigma^{2}} \Phi\left(-d_{0}\right)+\lambda^{2 \alpha / \sigma^{2}} \Phi\left(-d_{0}-2 f \sqrt{T-t} / \sigma\right) \quad \text { for } S>L
\end{array}\right.
$$

where

$$
d_{0}=\frac{\log \lambda-f(T-t)}{\sigma \sqrt{T-t}}
$$

and $f=\sqrt{b^{2}+2 r \sigma^{2}}, \quad b=-\left(r-\delta-\sigma^{2} / 2\right), \quad \phi=(b-f) / 2, \alpha=(b+f) / 2$ and $\lambda=S / L$.

Now if we take some integrable function $G$, then we compute the following expectations as

$$
\mathrm{E}_{t}\left[e^{-r\left(\tau_{L} \wedge T-t\right)} G\left(S_{T}\right) 1_{\left\{\tau_{L} \geq T\right\}}\right]=\left\{\begin{array}{l}
e^{-r(T-t)} \int_{0}^{L} G(x) u(x, t, T) d x \quad \text { for } S<L \\
e^{-r(T-t)} \int_{L}^{+\infty} G(x) u(x, t, T) d x \quad \text { for } S>L
\end{array}\right.
$$

where

$$
\begin{aligned}
& u(x, t, T)=\frac{\varphi\left(d^{-}(x)\right)-\lambda^{1-2(r-\delta) / \sigma^{2}} \varphi\left(d^{+}(x)\right)}{x \sigma \sqrt{T-t}} \\
& d^{ \pm}(x)=\frac{ \pm \log \lambda+\log (x / L)+b(T-t)}{\sigma \sqrt{T-t}} .
\end{aligned}
$$

Proof. The proof can be found in the Appendix of Broadie and Detemple (1995).

\section{References}

[1] Boyle, P. and Turnbull, S. (1989) Pricing and hedging capped options. J. Futures Markets 9 (41-54).

[2] Broadie, M. and Detemple, J. (1995) American capped call options on dividendpaying assets. Rev. Financ. Stud. 8 (161-191). 
[3] Chance, D. (1994). The pricing and hedging of limited exercise caps and spreads. Journal of Financial Research 17 (561-584).

[4] Detemple, J. (2006). American-Style Derivatives. Chapman \& Hall/CRC.

[5] Flesacker, B. (1992). The design and valuation of capped stock index options. Working Paper, University of Illinois at Urbana-Champaign.

[6] Karatzas, I. (1988). On the pricing of American options. Appl. Math. Optim. 17 (37-60).

[7] Palczewski, J. and Stettner, L. (2010). Finite horizon optimal stopping of discontinuous functionals with applications to impulse control with delay. SIAM J. Control Optim. 48 (4874-4909).

[8] Peskir, G. (2005). A change-of-variable formula with local time on curves. J. Theoret. Probab. 18 (499-535).

[9] Peskir, G. and Shiryaev, A. N. (2006). Optimal Stopping and Free-Boundary Problems. Lectures in Mathematics, ETH Zürich, Birkhäuser.

[10] QIu, S. (2016). American eagle options. Research Report No. 1 (2016), Probab. Statist. Group Manchester (45 pp). 